Modelling

Elsevier Editorial System(tm) for Ecological

Manuscript Draft

Manuscript Number: ECOMOD-18-694R2

Title: Consequences of neglecting cryptic life stages from demographic models

Article Type: VSI:Matrix Population Models

Keywords: Comparative demography; cryptic life stages; matrix population models; plant population ecology; seed bank; seed dormancy

Corresponding Author: Dr. Vuong Nguyen, Ph.D.

Corresponding Author's Institution: The Save Sight Institute

First Author: Vuong Nguyen, Ph.D.

Order of Authors: Vuong Nguyen, Ph.D.; Yvonne Buckley; Roberto SalgueroGomez; Glenda Wardle 


\section{Vuong Nguyen}

3 October 2018

Dear Prof Brian Fath and editorial board of Ecological Modelling,

Please find attached our manuscript entitled "Consequences of continuing to exclude cryptic life stages from demographic models" which we are submitting in consideration for publication in Ecological Modelling as part of the special issue on "Theory and Practice in Matrix Population Modelling".

This work identifies a methodological oversight that continues to plague the world of demographic matrix modelling and believe that it will be a perfect fit for the upcoming special issue. The findings are an 'inconvenient truth' for modellers and empirical demographers alike and we are candidly admitting that the work has been subject to peer review already. Reviewers were complementary about the importance of the topic and the clarity of the writing but held divergent view on the modelling based on their philosophical point of view. We provide substantial supplementary material to ensure that our methodology and analyses can be thoroughly checked.

In this manuscript, we explore the impact of excluding cryptic life stages stage from demographic models, focusing specifically on dormant seed banks as a common example of such a stage. We conducted a review of published plant matrix population models from 2008-2011 and found $47 \%$ had excluded the seed bank stage without providing justification; despite a call made over a decade ago for a better justification of seedbank exclusion (Doak et al, 2002), the field has not changed in this regard. This finding provided the appropriate motivation to investigate how this exclusion, and subsequent uncertainty in demographic parameters for the seed bank, will affect the model outcomes. We used a combined simulation and empirical approach, targeting published population models in which we found the study species had the potential for seed dormancy but the author(s) excluded the seed bank without justification, and (ii) ran Monte Carlo simulations with both uninformed and informed priors for seed bank vital rates based on literature estimates. Subsequently, we found that the relative importance of the seed bank to the long-term stochastic performance of the populations is context-specific, depending on the species and types of environmental cues present. Demographic data on the seed bank can be difficult to obtain, and our combined use of simulations and external information from the literature to assess the potential for dormancy and identify priors for vital rates provides a useful starting point for assessing whether a seed bank can be excluded without consequence or is actually important.

Yours sincerely, Vuong Nguyen 


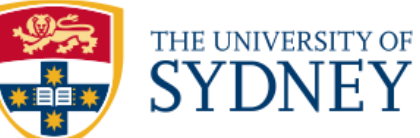

On behalf of the authors 


\section{Point by point response form}

E.1) I appreciate your positive responses to the numerous Reviewer's remarks in the form of a 12-page Table, yet I'm quite surprised that you have not written any word of gratitude to acknowledge Reviewer's 262 (!) 'PDF' Comments made in your original ms. By the way, the probability density function should better be abbreviated as 'p.d.f.'

Response: We thank the editor for reminding us of this oversight and we have acknowledged the anonymous reviewer for their substantial edits/comments. The abbreviation for probability density function has also been edited to p.d.f. as recommended.

E.2) The Highlight: 'Uncertainty in seed bank vital rates the major driver influencing model predictions' lacks the predicate and looks too hopeless. It should better be changed to something like: >Inclusion of the seed bank may change the model predictions made otherwise.<

Response: We have changed the highlight as recommended by the editor.

E.3) You give a misleading comment on ergodicity in lines 220-221: 'the condition that it is possible to transit from each stage to all other stages of the life cycle graph' In fact, this condition is called strong connectedness of the directed graph, while the ergodicity, as Caswell defined it following Cohen (1979), is its consequence. Also, the life cycle graph has no 'stages' - just nodes and arcs between them.

Response: We have corrected these term s to "strong connectedness" and "nodes" where appropriate.

E.4) The next sentence (222) should better be given in Present Indefinite. There still are other places where the Past Indefinite verb about the current study is neighbouring with refs to others, thus invoking ambiguity.

Response: We have read through the manuscript and fixed further instances of the past indefinite verb being used to describe the current study and the outcomes.

E.5) There other places in English, too, to be corrected, in particular, wrong commas (265) and missed commas/full stops after displayed formulae.

Response: Commas have been added/removed where appropriate

E.6) Reviewer's point 6.4) (about elasticity matrices) has been well responded in Revision 1, but Table $S 2$ lacks an explanation of what $\varnothing$ means. 
Response: We have included the following the caption for Table S2 "The $\varnothing$ symbol indictates that the elasticity is not defined for the corresponding element of the plant matrix model since the link was not present in the life cycle graph."

E.7) The correctness of notation $\lambda 1$ is accompanied with its greater vulnerability to misprints than just $\lambda$, such as $\lambda 1(402,430$, elsewhere) or $\lambda 1$ (253), hence it needs greater care.

Response: Notation for all instances of $\lambda$ has been checked for correct use of superscripts and italicisation. 


\section{Consequences of neglecting cryptic life stages from demographic models}

2

3 Vuong Nguyen ${ }^{1 *}$, Yvonne M. Buckley ${ }^{2,3}$, Roberto Salguero-Gómez ${ }^{2,4,5}$ and Glenda M. Wardle ${ }^{1}$

4

$5 \quad{ }^{1}$ Desert Ecology Research Group, School of Life and Environmental Sciences, The University of

6 Sydney, Sydney, NSW 2006, Australia

$7 \quad{ }^{2}$ School of Biological Sciences, The University of Queensland, Queensland, 4072, Australia

$8 \quad{ }^{3}$ School of Natural Sciences, Department of Zoology, Trinity College Dublin, Dublin, Ireland

$9 \quad{ }^{4}$ Evolutionary Biodemography Laboratory, Max Planck Institute for Demographic Research,

10 Rostock 18057, Germany

$11{ }^{5}$ Department of Zoology, University of Oxford, Oxford, UK

12

$13 *$ Correspondence author. E-mail: phuc.nguyen@sydney.edu.au 


\section{Abstract}

Information on individuals from all stages of life is crucial to explore their ecology, evolution and conservation biology. However, the life cycles of many species contain cryptic life stages that are difficult to detect and track over time and are therefore omitted from demographic models. One example is the dormant seed bank, an evolutionary bet-hedging mechanism that buffers plant populations in variable environments. To evaluate this methodological oversight, we conducted simulations to explore the effect of seed bank parameter uncertainties on demographic outputs such as the deterministic $\left(\lambda_{1}\right)$ and stochastic population growth rate $\left(\lambda_{\mathrm{S}}\right)$, and extinction probabilities of 12 plant species. We have used uninformed and informed priors for seed bank parameters based on literature estimates, and reconstructed published models in which the seed bank was excluded without justification. Trials on removing the seed stage from models (6 species) explored the worst-case scenario for ignoring the seed bank. Inclusion of a seed bank and demographic uncertainty in seed bank parameters have little impact on stable populations $\left(\lambda_{1} \approx 1\right)$ with high post-seedling survival. When populations deviate from stability or demonstrate temporal demographic variation, greater changes in $\lambda_{1}$ and the range of possible growth rates caused by demographic uncertainty are observed. As expected, decreasing populations $\left(\lambda_{1}<1\right)$ benefit from the inclusion of a seed bank through increases in the growth rate and extinction times, whereas increasing populations $\left(\lambda_{1}>1\right)$ are slowed down. While germination estimates from the literature cannot accurately reflect those obtained in the field, they provide a starting point to assess the relative importance of a seed bank. The exclusion of the seed bank must be justified by confirming that dormancy is either non-existent or not important. Accounting for cryptic stages in demographic models will produce better informed management decisions for threatened or invasive species. 
39 Keywords: Comparative demography, cryptic life stages, matrix population models, plant 40 population ecology, seed bank, seed dormancy.

\section{Highlights}

- Inclusion of seed bank reduced extinction times and improved long-term persistence

- Published literature and simulations can assess the importance of the seed bank

- Uncertainty in seed bank vital rates the major driver influencing model 


\section{Introduction}

Demographers have long recognised that individuals in a population span across different stages of their life cycle, from propagules up to reproductive adults, and that these stages contribute to its persistence in different ways - failing to account for all these stages can therefore be misleading (Caswell, 2001). However, the life cycles of many species contain cryptic life stages that may be difficult to detect or sample. These cryptic life stages occur over a range of taxonomic groups, and may include hibernating or dormant individuals, clonal individuals, eggs, mobile propagules, or simply stages occupying inaccessible locations (Geiser, 2004, Katzner et al., 2011, Shefferson, 2009, Tanner, 2001). The dormant seed bank is perhaps one of the most common examples of the cryptic life stage whereby seeds enter prolonged dormancy ( $>1$ year) within the soil or above ground in woody cones, and can make up a significant portion of the population (Baskin and Baskin, 2014, Harper, 1977). By spreading germination through time, seed dormancy acts as a bet-hedging mechanism mitigating the effects of unfavourable years in exchange for reduced proliferation during favourable years (Gremer et al., 2012, Venable, 2007). In extreme cases, seed banks can prevent local extinctions when above-ground populations are killed off entirely (Stöcklin and Fischer, 1999). Seed dormancy has also been shown to promote species co-existence in variable environments (Warner and Chesson, 1985) and act as a temporal reservoir for genetic diversity (Vitalis et al., 2004).

With such substantial consequences for population dynamics, it would be a major oversight to exclude the seed bank, or any cryptic life stage, from population estimates and demographic analyses. This argument is indeed not new: the need to incorporate the seed bank into demographic models has been recognised for decades (Harper, 1977, Kalisz and McPeek, 1992). Matrix population models, which divide individuals into their respective stage classes, are 
the most common tool for modelling plant populations - the online database of plant matrix models, COMPADRE v5.0.0 contains 8,906 published matrices for 759 species ( COMPADRE Plant Matrix Database, v5.0.0., 2019, Salguero-Gómez et al., 2015). These models have a long history in the management of threatened and invasive species (Crone et al., 2011). Yet, a previous review of 70 published matrix population models found almost half (43\%) had excluded the seed bank stage without justification (Doak et al., 2002). Surprisingly, we have found that this proportion has remained unchanged in the last decade (47\%; Table 1). We acknowledge that including the seed bank is not straightforward as the estimation of vital rates such as survival and germination generally rely on additional laboratory experiments (Crone et al., 2011), and even these would inflate actual in situ estimates (Gross and Mackay, 2014).

Furthermore, unless such experiments are rigorous enough to include age-specific vital rates (Kalisz and McPeek, 1992), the seed bank has been generally assumed to be unstructured. Excluding the seed bank may be justified if it is discovered the species, or population, does not possess dormant seeds (see Baskin and Baskin, 2014 for review on the presence of seed dormancy), or if it is mathematically and empirically justified in a modified model (Logofet et al. 2017), however, these cases are the minority compared to those that do not provide justification (Doak et al., 2002; Table 1). Unjustified exclusion can have real-world consequences if, for example, the seed bank of an invasive species recolonises after the adult individuals have been eliminated (Regan et al., 2011), or if the population size and persistence of threatened species is underestimated (Doak et al., 2002).

The practical issues of including the seed bank and its exclusion from demographic models raise two important questions. First, how can we bridge the gap in our understanding of whether a seed bank is necessary to adequately model the population dynamics in the absence of 
field data? Second, how does uncertainty in the presence of a seed stage and its transition rates translate into uncertainty in the model? Addressing these questions using a simulation-based approach can be useful for investigating a range of scenarios and assumptions but may not be biologically meaningful without application to real-world examples. An empirical approach, on the other hand, may be limited in its scope if it were to focus on a single case study, as there are clear difficulties in obtaining individual-level records for published models. Using published matrix models that have excluded the seed bank without justification and information on dormancy and germination rates from the literature, we implement a combination of these approaches and perform a comparative analysis across a range of species and life forms (12 species including eight trees, three shrubs and one herb). We conduct Bayesian Monte Carlo simulations on these published models using uninformed and informed priors (based on literature estimates) for seed bank vital rates to gauge the level of uncertainty when the seed bank presence and parameters are unknown. To complement these addition simulations, we also investigate the implications of assuming the absence of a persistent seed bank by intentionally removing the seed bank from models which did include it.

We make a number of predictions based on the evolutionary advantages of seed dormancy. First, we might expect the relative importance of seed banks to be greater for species in unpredictable environments with variable post-seedling survival compared to stable populations of long-lived species with consistently high adult survival (Venable, 2007).

Furthermore, the deterministic growth rate $\left(\lambda_{1}\right)$, which assumes vital rates remain constant, should increase during poor demographic years $\left(\lambda_{1}<1\right)$ and decrease during productive years $\left(\lambda_{1}\right.$ $>1)$ to reflect the function of the seed bank as a bet hedging mechanism against unfavourable years (Gremer et al., 2012, Kalisz and McPeek, 1992). Similarly, elasticities (the relative 
influence of each demographic process to the population growth rate; de Kroon et al., 2000) for seed bank transition rates should be greater during poor years. We should also observe increases in times to local extinction and stochastic growth rates $\left(\lambda_{\mathrm{S}}\right)$, which incorporate temporal variation in vital rates. Using both an empirical and simulation-based approach, we provide more robust projections of the demographic fates of populations and help to motivate the inclusion of the seed bank into demographic models.

\section{Materials and Methods}

\section{Database of population matrix models}

At the time this study was conducted, the COMPADRE Plant Matrix Database had not yet become open-access online (Salguero-Gómez et al., 2015), with the most recent update consisting of 204 terrestrial plant species up to 2007, which we hereafter refer to as the "MPM2007" database constructed by the Vegetation Function Working Group (see Burns et al., 2010 for further information). We have used the ISI Web of Science to identify published plant matrix population models from 2008-2011, which we hereafter referred to as the "MPM20082011" database.; These studies which-would later go on to be added to the COMPADRE $\underline{\text { database. }}$. Examples of keywords used in our search include "matrix model*", "transition matrix", "demographic model*”, "population model*”, "population viability analysis", "population growth rate", and "population dynamics". The addition of * at the end of a search term acts as a wildcard and allows for alternative forms of a given word, e.g. model(s), model(ling). We have found 55 studies with matrix models for 77 terrestrial plant species including 38 trees, 27 herbs, 8 shrubs and 4 succulents.

\section{Confirmation of the seed bank}


Using Baskin and Baskin (2014) and the species name as a search term in ISI Web of Science, we have verified whether a seed bank was reported for species in which seed bank exclusion was unjustified (by not mentioning the seed bank; Table 1). While each population may not necessarily possess a seed bank, we can still determine the impact of its presence on model outcomes. In total, we have constructed new matrix population models for the 12 plant species for which published germination data on either the species or the genus were available and the original matrices were provided (see Supplementary File S1 for all original and reconstructed matrices).

\section{Seed germination submodel}

The seed bank may be modelled as structured or unstructured. In the unstructured seed bank model, it is assumed that the seed bank does not age and is potentially immortal. In the structured seed bank model, the seed bank progressively ages, with potentially different survival, viability and germination rates, until it reaches time step $n_{2}$ at which point the vital rates remain constant or the seeds die if they do not germinate (see Figure 1B for a life cycle example of the structured seed bank and $1 \mathrm{C}$ for the unstructured seed bank). The maximum length of time the seed bank can remain viable is variable across species and is difficult to quantify - some seeds have been documented germinate after centuries of dormancy (Fenner, 2000).

\section{Addition and removal of seed bank stages}

Adding, removing, or excluding stages without justification alters the dimensionality of the model, and this will affect the demographic parameter estimates. Merging or further dividing up stage classes directly alters vital rates for each class being affected and the speed of the life cycle, where decreasing the dimensionality reduces the number of stages to progress through the 
life cycle and vice versa (Salguero-Gómez and Plotkin, 2010). As the seed bank functions as a bet hedging mechanism, delayed movement through the life cycle is to be expected and this is reflected in our hypotheses. Since the seed bank is a separate, discrete stage class that involves no splitting or merging, transiation rates for the other classes should remain unaffected by its inclusion. However, seedling fecundity would need to be lowered accordingly to account for observed seedlings emerging from the seed bank to avoid overestimating fecundity. We use sSeedling counts une to estimate fecundity in all models where the seed bank was excluded without justification. For simplicity, we have-assumed no clonal reproduction into the seedling stage such that the observed seedlings only consist of two components: the individuals that germinated immediately between year $t$ and $t+1$ and those that germinated from the dormant seed bank from prior years. The number of observed seedlings can thus be described by the following equation:

$$
f_{i, j}=F_{j}(t) v g s+F_{j}(t-1) v(1-g)(1-d) g_{b 1}+\mathrm{F}_{j}(t-2) \mathrm{v}(1-g)\left(1-g_{b 1}\right)(1-d)^{2} g_{b 2}+\ldots,
$$

where the first term on the right hand side represents immediate germinants, the second term represents germination of seeds from the seed bank produced at the previous time step, and the third term represents germination of seeds from the seed bank produced two time steps ago, and so on. Notations:

$f_{i, j}=$ per-capita fecundity into stage class $i$ by stage class $j$ in one time step; $F_{j}(t)=$ total fecundity by stage class $j$ at time $t$; $v=$ seed viability rate during the step $t$ to $t+1$; $g=$ non-seed bank germination rate at the period $t$ to $t+1$; $g_{b l}=$ germination rate from seeds that remained dormant in the seed bank for 1 time step; 
$g_{b 2}=$ germination rate from seeds that remained dormant in the seed bank for 2 time steps; $s=$ seedling survival rate until the next census; $d=$ seed bank mortality rate

See Fig. 1 for more information.

To simplify the inclusion of the seed bank stage under limited information, we have made the following assumptions. First, the seedling survival rate $(s)$ from the period $t$ to $t+1$, is equal to the seed bank survival rate $(1-d)$. Second, we start with simulations that focus on the contribution of the seed bank from $t-1$. This unstructured submodel captures the predominant seed bank contribution since the contribution of seeds from previous years will diminish exponentially with each year; if we assume the vital rates to be not seed age dependent. Alternative age-structured formulations are explored below. Additionally, we assume the fecundity from the previous year assumed-to equal the fecundity in the current year, i.e. $F_{j}(t)$ $=F_{j}(t-1)$, hereafter denoted by $F_{j}$. Finally, the probability of germinating within the census year was-is equal to the probability of germinating from the seed bank, i.e. $g=g_{b}$ (Kalisz and McPeek, 1992). Using the published information on germination, we simplify Eq. 1:

$$
f_{i, j}=F_{j} v g s+F_{j} v(1-g)(1-d) g
$$

where the first term on the right-hand side represents the number of seedlings germinating immediately between $t$ and $t+1$, and the second term corresponds to germination from the seed bank. For estimates of the deterministic and stochastic growth rates, we set seed viability $(v)$, seedling survival $(s)$ and seed survival $(1-d)$ equal to 1 unless this information was provided in the literature alongside germination estimates. Monte Carlo simulations (described below) then 
investigated drawing these values from both uninformed and informed prior distributions. Taking the observed $f_{i, j}$ from the original matrix, we solve Eq. 2 for $F_{j}$ and calculate the per-capita fertility contributions to seedlings and seed bank (See Supplementary Method S1 for an example). Following the same approach, we also explore alternative model formulations and the effect of different assumptions but only performed Monte Carlo simulations for the unstructured scenario, which is the simplest and most common approach to including the seed bank. The alternatives include the addition of an age-structured seed bank distinguishing seeds from $t$ - 2 and $t$ - 3 and more conservative estimates on the effect of the seed bank by further assuming the seeds do not survive beyond the first, second or third year (See Supplementary Method S1 and Table S1 for the alternative formulations and population growth rates for all models).

Researchers excluding the seed bank from a model make the implicit assumption that it is not present in the population. Thus we intentionally remove the seed bank from selected models to explore the implications of this assumption. Given the large number of potential models to choose from, we have decided to focus on cases in which the seed bank was known to be important (elasticities of $\lambda_{1}$ to seed survival, germination or fecundity $>0.1$ ) to demonstrate the worst case scenario that might be possible when the seed bank is ignored. We have identified six such species from the MPM2007 and MPM2008-2011 databases that fulfil this criteria whilst retaining ergodicitystrong connectedness, the condition that it is possible to transit from each stage-node to all other stages-nodes of the life cycle graph (Caswell, 2001), such as those in Figure 1. We use finformation on seed viability, seedling survival, and seed survival eredif provided, but were-otherwise we set them to 1 , and we no longer assume that $g=g_{b}$ since $g_{b}$ can be obtained from the original matrix. We estimate $t$ The rate of seed germination within the studied period, $g$, was then estimated-based on partitioning the total reproductive output (Eqs. 1 
and 2) to recalculate only the fecundity into the seedling stage. To further ensure that no artefacts

from changes to reproduction or mortality are introduced, the removal protocol the reverse of the addition protocol, , and and we -check ef that the recovery of the initial matrix after these manipulations was performedcan be recovered by reapplying the addition protocol (See Supplementary Method S2 for an example).

\section{Analyses}

\section{We use R (v 3.1.1; The R Foundation for Statistical Computing) to calculate Ppopulation growth} rates $\left(\lambda_{1}\right)$, elasticities, stochastic simulations exploring temporal variation in vital rates and Monte Carlo simulations exploring uncertainty in seed bank vital rates (were calculated using $\mathrm{R}$ ( $v$ 3.1.1; The R Foundation for Statistical Computing; see Supplementary File S2 for code to perform Monte Carlo simulations).

$$
\text { Monte Carlo simulations used anWe use uninformed priors for germination, viability, }
$$
seedling survival and seed survival for Monte Carlo simulations by drawing these ratesand thus these rates were drawn from a uniform distribution for the simulations. Although we might expect covariance among vital rates for the below- and above-ground individuals (Morris and Doak, 2002), introducing this structure is complicated particularly when dealing with the seed bank. For example, models that do include the seed bank generally estimate seed bank vital rates through separate germination experiments such that the covariance between the seed bank and the above-ground population is lost in the process. While the impact of this covariance structure on the model outcomes would be of interest, we instead focus here on the exclusion of the seed bank from demographic models and, leaveing investigations regarding correlation structure for future research. 
for each set of parameters, and report the mean growth rate with confidence intervals obtained by the $2.5 \%$ and $97.5 \%$ quantiles. We then determine the effect of using informed priors via literature estimates for germination, and occasionally viability when available, on the resulting probability density function (PDFp.d.f.) of the distribution of growth rates. Publications on germination often contain multiple treatments and an estimated mean germination rate for each treatment. We have specified our prior distributions depending on the type of demographic year to reflect the function of the seed bank as a bet hedging mechanism (the highest germination rate available when $\lambda_{1}>1$ and the lowest when $\lambda_{1}<1$; Gremer et al., 2012, Kalisz and McPeek, 1992). However, if only one germination rate was provided, we use this value for all population matrix models regardless of the demographic year. If only the mean matrix was provided (arithmetic mean matrix across all years and sites), we use the average germination over all treatments. We model both germination and viability using the beta distribution as it varies

between 0 and 1 , with the distribution mode equal to the germination rate. We have-also calculated $\lambda_{1}$ for fixed values of the germination rate (0.01-1 by increments of 0.01$)$ and viability rate $(0.1,0.5$, and 1$)$ to capture the total range of possible values when only these parameters are allowed to vary. The matrices in which the germination rate was set to that obtained from the literature were $\underline{\text { are }}$ used to calculate single point estimates of $\lambda_{1}$, elasticity matrices, $\lambda_{\mathrm{S}}$ and extinction probability, with viability, seed survival and seedling survival set to 1 if no information was available. The initial population for all stochastic and extinction probability calculations hereafter consists of 1,000 individuals at the stable stage distribution, calculated as the normalized dominant right eigenvector (Caswell, 2001) of the mean matrix. We have

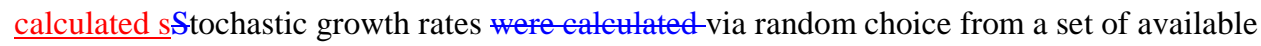

Formatted: Font: Italic

Formatted: Subscript

Formatted: Font: Italic

Formatted: Subscript 
matrices (Kaye and Pyke, 2003), where each matrix has an equal probability of being selected at each time step. The initial population 5,000 omitted to remove transient dynamics (Kaye and Pyke, 2003, Morris and Doak, 2002). We use Tthe mean and variance of the growth rates were used to obtain an estimate for $\lambda_{\mathrm{s}}$ and $95 \%$ confidence intervals.

We also compare extinction probabilities between models with and without seed banks.

Times to extinction (defined as population size $\leq 1$ individual) were $\underline{\text { are }}$ calculated for matrices predicting population decline. We project an initial population for individual matrices over 100 years and compared the difference in times to extinction with and without the seed bank to quantify the effect of the seed bank on population persistence. We then conduct stochastic simulations to calculate the cumulative probability of quasi-extinction (Morris and Doak, 2002) with a quasi-extinction threshold set to 100 individuals (i.e. one tenth of the initial population size). We perform 100 runs, where a single run consists of 5000 iterations each projected over 100 years, and calculate the mean fraction of projections that fell below the quasi-extinction threshold during or before the year $t$ (Morris and Doak, 2002).

\section{Results}

Addition of the seed bank and demographic uncertainty

Inclusion of an unstructured seed bank showed fairly small differences in $\lambda$ for most species regardless of the assumptions made (See Fig. 2 and Table 2 to compare the growth rates).

However, the potential range of growth rates when demographic parameters are uncertain can be large depending on the species and the amount of temporal variation observed. The 8 tree species $_{2}$ for example, haved stable populations $\left(\lambda_{1} \approx 1\right)$; and variation in seed bank parameters has $\mathrm{d}$ little influence in all of the models with the exception of Illicium anisatum 
(Schisandraceae). Subsequently, using informed priors for these species hads little impact on the PDF-p.d.f. of the distribution of possible growth rates. In contrast, the shorter-lived shrub species, Atriplex acanthocarpa (Amaranthaceae) and Sambucus racemosa (Adoxaceae), were-are more sensitive to the introduction of a seed bank and demographic uncertainty in their vital rates. Here, using an informed prior on germination narroweds the PDF-p.d.f. of the distribution of possible growth rates for the A. acanthocarpa 1996-1997 matrix during an extremely productive year (Fig. 3). Conversely, the use of prior information during the poorer 1997-1999 years appeareds to increase the spread of possible growth rates. Despite this, poor years tend to have a narrower distribution of possible growth rates and are therefore less sensitive to changes in demographic parameters compared to good years (Figs. 3 and 4). Generally, the PDF-p.d.f. of growth rates wereare skewed, with peaks being concentrated when germination rates wereare high and greater variation in $\lambda_{1}$ when germination is low (Figs. 3 and 4). However, we note these simulations only investigated the unstructured seed bank scenario. Further uncertainties exist with regards to the longevity and structure of the seed bank, both of which were only briefly explored in this study (Supplementary Table S1).

Elasticity analyses in most plant species, particularly the trees, revealed survival of the adult or late juvenile stages to be the most important demographic process affecting $\lambda_{1}$. The addition of the seed bank did not change this pattern except in the case of A. acanthocarpa during 1997-98 and 1998-99 when the populations were declining (Supplementary Table S2). In the $1997-98$ period, the elasticity of $\lambda_{1}$ to stasis in the third adult stage (0.5759 to 0.0647$)$ following the inclusion of the seed bank, whereas the elasticity of $\lambda_{1}$ to stasis in the seed bank was high (0.7919). Similarly, the elasticity of $\lambda_{1}$ to stasis in the first adult 
stage during the $1998-99$ period, was-is reduced from 0.3983 to 0.12 after seed bank addition,

while the elasticity of $\lambda_{1}$ to survival in the seed bank was high (0.5039).

\section{Removal of the seed bank and uncertainty in seed bank presence}

Removal of the seed bank led to-further increases population increase-growth when $\lambda_{1}>1$ since germination is no longer delayed, and toreduces d population persistence when $\lambda_{1}<1$ since the buffering effect is no longer present (Table 3). The notable exception is the wetland herb

Helenium virginicum (Asteraceae), which experiencedwhere removing the seed bank decreases-a eomparatively high decrease in $\lambda_{1}(1.1145$ to 0.6985$)$ when the seed bank was removedeven though the population was increasing. This unusual response is likely a combination of extremely high fecundity into the seed bank and within-the-seed-bank stasis rate (1025 and 0.987 respectively), and the comparatively low fecundity and survival of the smallest stage (0.0075 and 0.199 respectively; see Supplementary File S1 for the original and updated matrices). Decreases in $\lambda_{1}$ were is also particularly substantial for Carduus nutans (Asteraceae) and Digitalis purpurea

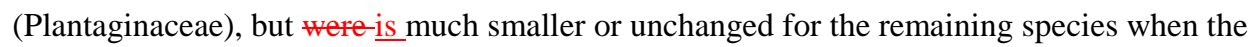
seed bank was-is removed for poor years.

\section{Extinction probabilities}

Inclusion of the seed bank using point estimates for germination generally resultsed in longer times to local extinction (Supplementary Figure S1). Extinction times for A. acanthocarpa for the 1997-98 and 1998-99 matrices receivedhave relatively high increases in extinction times (> 25 years) when the seed bank is included. In addition, certain populations of A. canescens and the perennial herb $D$. purpurea are no longer predicted to go extinct in the next 100 years. The populations of the short-lived herb $C$. nutans received-have comparatively smaller increases 
in extinction times. In one population, however, including the seed bank doubles the time to extinction from 8 to 16 years, which is relatively high when compared to the life expectancy of the species $\left(\eta_{E} \cong 2-3\right.$ years, based on the fundamental matrix estimation; Caswell, 2001).

The seed bank generally improves of extinction probability, but patterns varyied across species (Supplementary Figure S2). The cumulative probability of extinction at 100 years decreaseds drastically for A. acanthocarpa from 0.19 to 0.01 following inclusion of the seed bank. The extinction probability for $A$. canescens showed-has an interesting pattern where the matrix model with the seed bank results initially in a higher extinction probability than the original in the first 38 years and eventually in a lower extinction probability, but in the remaining two cases, the seed bank has $d$ little effect on the extinction probability. Both populations of $D$. purpurea within 100 years. One population received $\mathrm{a}_{2}$, however, there is a modest increase in extinction time (16 to 30 years) for one population and while the increase was-a smaller increase in extinction time for the other (4 to 9 years).

\section{Discussion}

The presence of cryptic life stages and their exclusion from demographic models continues to be an impediment to understand and model the population dynamics. These cryptic stages are diverse, ranging from clonality, vegetative dormancy, hibernation, diapause, aestivation, etc., as is their potential impact on population dynamics and model outcomes. Using the seed bank as a well-known example of a cryptic life stage, we demonstrate the use of simulations as a means to reduce uncertainty in seed bank dynamics and to reveal the relative importance of the seed bank and its effect on model outcomes. Ignoring the seed bank in a population can produce variable degrees of uncertainty in the estimates of growth rates and extinction probabilities depending on 
the observed temporal variation in key vital rates. For example, models with high seedling survival and low temporal variation in demographic parameters were $\underline{\text { are }}$ more robust to seed bank uncertainty compared to models with high temporal variation in growth rates and demographic parameters. Furthermore, the potential impact of the seed bank can be influenced by the assumptions underpinning the models and the accuracy of the vital rates that are obtained.

\section{Dealing with the seed bank when its presence is unknown or uncertain}

Anticipating the species for which the seed bank will be an important factor is challenging due to the uncertainty in the demographic parameters of the seed bank stage across species, populations and time. Here, we provide a protocol outlining the different approaches one can take when conducting a plant population study (Fig. 5). First, the potential presence of a seed bank can be determined through a literature search of seed germination and dormancy values (Baskin and Baskin, 2014, is of particular note). Second, if a seed bank in the population is confirmed, the best option would be to obtain field data over the study period to provide estimates for the seed bank vital rates. Without this field data, characterising the potential role of the seed bank becomes exceptionally difficult and assumptions regarding fecundity, seed survival, viability and longevity need to be made. Prior knowledge from the literature can narrow down these possibilities to some extent, although seed characteristics and seed age composition can vary substantially between sites. Also, knowledge from the literature or from field and glasshouse trails may not necessarily reflect those of the study site and must therefore be interpreted with caution (Gross and Mackay, 2014). Finally, simulations can be used to complement these literature estimates to efficiently investigate the numerous possible assumptions regarding seed bank vital rates and determine plausible effects of a potential seed bank. Garcia et al. (2010), for example, performed simulations covering feasible information obtained in the field or from other 
publications for the long-lived orchid Cypripedium calceolus (Orchidaceae) to justify excluding the seed bank from their models. Likewise, for many of our species, without the benefit of greater observed temporal variation, ignoring the seed bank would appear to have beenbe of minimal consequence for the original study, but it is still crucial that an investigation is performed to provide this justification. Otherwise, effective implementation and assessment of management strategies will be hindered as the demographic model is potentially missing a key component of the life cycle.

\section{Uncertainty in the seed bank and its effect on model outcomes}

Demographic uncertainty in the estimation of vital rates appears to be the major driver in influencing the model predictions but varies by species. For example, 1 Long-lived tree species were are virtually unaffected by both the inclusion of the seed bank; and variation in their demographic parameters. This is not entirely surprising as high adult survival and reduced mortality when environmental conditions are poor can be an alternative strategy to improve the long-term fitness (Clauss and Venable, 2000). In such species, it is likely that the seed bank will be of little consequence, although it would still be prudent to confirm this suspicion (e.g., using simulations) before ignoring the seed stage. Additionally, this does not necessarily mean the seed bank will be unimportant if the population were to experience a large disturbance. In contrast, in populations experiencing greater fluctuations in demographic parameters and population growth rates, the contribution of the seed bank is more visible as is evident for A. acanthocarpa, where previously high elasticities for juvenile and adult survival sere shiftedshift towards survival in the seed bank during poor demographic years (Table S2).

Temporal variation and the type of demographic year are also factors that will affect of demographic uncertainty. Alterations in germination and viability rates during poor demographic 
years $\left(\lambda_{1}<1\right)$ haved relatively little impact on the growth rate compared to favourable years

411 when $\lambda_{1}$ exceeded 1 (Fig. 4). Large reproductive failures during poor years would have resulted in low seed bank and seedling input, reducing the impact of varying seed bank vital rates on $\lambda_{1}$.

413 In contrast, we would expect variations in germination and viability rates during poor years to

414 have a much larger impact on long-term persistence and environmental stochasticity.

415 Nonetheless, with the inclusion of prior information from the literature, the PDF-p.d.f. of the

416 distribution of growth rates during these poor demographic years shifts towards one (Fig. 3). We

417 note however that this is assuming dormancy is higher (low germination) during poor

418 demographic years and lower during productive years (Kalisz and McPeek, 1992), but seed

419 dormancy needs not necessarily be correlated with fitness. Interestingly, the use of literature did not necessarily reduce uncertainty in the range of possible growth rates and in fact becomes more variable when dormancy is high (Fig. 3). This is an important result as an uninformed simulation may underestimate the level of uncertainty in demographic output if prior information suggests potentially high levels of dormancy. In contrast, when dormancy is suspected to be low, the impact of the seed bank will not be as great, thus variation in its demographic parameters will be of much less consequence.

In addition to variations in demographic parameters, changes to how the seed bank is included can impact the model structure and outcomes. Our uncertainty simulations investigate a 1-year seed bank as a counterfactual example to illustrate the need for including a seed bank. We acknowledge that while single point estimates of $\lambda_{1}$ under the unstructured scenario showed minor increases compared to the original models, this gradually reduces when additional age classes are introduced (See Table S1 to compare the growth rates). This is likely because a

Formatted: Font: Not Italic 
produced in previous years, thus compounding the effect of delayed germination. Similarly, if we assume seeds do not survive beyond the first, second or third year, the effect of the seed bank is almost negligible (See Table S1). However, such estimates are overly conservative and most models that do include the seed bank do not make this assumption.

Another interesting scenario that can be tested is that of no seed bank $k_{-}$, and $\Theta \underline{\text { Our removal }}$ simulations provides insights into the worst-case scenarios when the seed bank is ignored despite being crucial to persistence (as indicated by high elasticities to seed bank transitions). As expected, the implications of ignoring the seed bank in these cases were generally much more evident; with decreases in $\lambda_{1}$ of up to 0.4 , although these reflect the worst-case scenarios and changes in $\lambda_{1}$ would not generally be so dramatic. The assumption of fecundity being equal to

Formatted: Font: Not Italic that of the previous year $\left(F_{j}(t)=F_{j}(t)\right)$ was necessary due to lack of information, however, interannual variation is likely to play an important role for many species. When variance in the fecundity is high, the contribution of the seed bank would be overestimated in good demographic years and underestimated in bad demographic years. This would likely have similar consequences on any changes in $\lambda_{1}$, thus we might expect the benefit of having a seed bank during poor years to also be underestimated.

Analysis of extinction probabilities over shorter time periods can provide additional insights not considered under asymptotic analyses. Comparing the difference in extinction times and cumulative extinction probabilities between models with and without the seed bank can provide an empirical measure of the uncertainty in population persistence when the seed bank is ignored, and this measure can vary substantially between years and species. Although increases in extinction times can appear trivial, they must also be considered in context of the life expectancy. For example, Tthe seed bank had little effect on the times to extinction for the 
populations of $C$. nutans for example. However, given the short life expectancy of this species, even a small increase in extinction time may prove crucial.

\section{Conclusions}

There is still much progress to be made with regards teconcerning the seed bank, and it is but one of many cryptic life stages with the potential to create biases in our model outputs. Adult plants, for example, can undergo vegetative dormancy, whereby individuals do not sprout above ground for one or more growing seasons, resulting in overestimated mortality rates and incorrect assignment of false birth events to re-emerging individuals (Logofet et al., 2019, Shefferson, 2009). Demographic studies on animals can be additionally problematic as certain stages of the life cycle may occupy inaccessible locations or be absent from sampling designs. Katzner et al. (2011) found raptor populations to be drastically underestimated as monitoring programs only captured the breeding portion of the population. In both plant and animal studies, incorrect estimates of population size, persistence, and occupancy in demographic models can result in suboptimal management decisions and make it difficult to assess the success of these management actions (Regan et al., 2011). While this studywe have focused exclusively on plant population models in this study, characterising the extent of exclusion of cryptic life stages in demographic models for animals is just as important and open for investigation with the availability of the COMADRE Animal Matrix Database (Salguero-Gómez et al., 2016).

\section{Acknowledgements}

The authors would like to acknowledge the critical review by an anonymous reviewer that has greatly improved the manuscript, thankE. Crone and J. Ehrlén for helpful comments in previous versions of this manuscript, and S. Ramula and members of the ARC-NZ Research Network for Vegetation Function Plant Population Syndromes working group. 
$480 \quad$ Author Contributions

481 The first and last author conceived and designed the study. The first author collected the data and 482 performed the analysis. All authors contributed to the study methodology and writing of the 483 manuscript. 
Abe S, Motai H, Tanaka H, Shibata M, Kominami Y, Nakashizuka T (2008). Population maintenance of the short-lived shrub Sambucus in a deciduous forest. Ecology, 89, 11551167.

Adams VM, Marsh DM, Knox JS (2005). Importance of the seed bank for population viability and population monitoring in a threatened wetland herb. Biol. Conserv., 124, 425-436.

Angert AL (2006). Demography of central and marginal populations of monkey flowers (Mimulus cardinalis and M. lewisii). Ecology, 87, 2014-2025.

Baskin CC, Baskin GM (2014). Seeds: Ecology, biogeography, and evolution of dormancy and germination, Academic Press, San Diego, California.

Burns JH, Blomberg SP, Crone EE, Ehrlén J, Knight TM, Pichancourt JP, Ramula S, Wardle GM, Buckley YM (2010). Empirical tests of life-history evolution theory using phylogenetic analysis of plant demography. J. Ecol., 98, 334-344.

Caswell H (2001). Matrix population models: construction, analysis and interpretation, Sinauer Associates, Inc., Sunderland, Massachusetts.

Chien PD, Zuidema PA, Nghia NH (2008). Conservation prospects for threatened Vietnamese tree species: results from a demographic study. Popul. Ecol., 50, 227-237.

Clauss MJ, Venable DL (2000). Seed germination in desert annuals: an empirical test of adaptive bet hedging. Am. Nat., 155, 168-186.

COMPADRE Plant Matrix Database. 2019. COMPADRE Plant Matrix Database v5.0.0. [Online]. Max Planck Institute for Demographic Research (Germany). [Accessed 20th May 2019].

Crone EE, Menges ES, Ellis MM, Bell T, Bierzychudek P, Ehrlén J, Kaye TN, Knight TM, Lesica P, Morris WF, Oostermeijer G, Quintana-Ascencio PF, Stanley A, Ticktin T, Valverde T, Williams JL (2011). How do plant ecologists use matrix population models? Ecol. Lett., 14, 1-8.

de Kroon H, Plaisier A, J.V. G (1987). Density dependent simulation of the population dynamics of a perennial grassland species, Hypochaeris radicata. Oikos, 50, 3-12.

de Kroon H, van Groenendael J, Ehrlen J (2000). Elasticities: a review of methods and model limitations. Ecology (Wash. D. C.), 81, 607-618.

Doak DF, Thomson D, Jules ES (2002). Population viability analysis for plants: understanding the demographic consequences of seed banks for population health. In: Beissinger SR, McCullough DR (eds.) Population Viability Analysis. The University of Chicago Press, Chicago, Illinois, USA.

Fenner M (2000). Seeds: the ecology of regeneration in plant communities, CABI Publishing, Wallingford, UK.

Garcia MB, Goni D, Guzman D (2010). Living at the edge: local versus positional factors in the long-term population dynamics of an endangered orchid. Conserv. Biol., 24, 1219-1229.

Geiser F (2004). Metabolic rate and body temperature reduction during hibernation and daily torpor. Annu. Rev. Physiol., 66, 239-274.

Gremer JR, Crone EE, Lesica P (2012). Are dormant plants hedging their bets? Demographic consequences of prolonged dormancy in variable environments. Am. Nat., 179, 315-327.

Gross C, Mackay D (2014). Two decades of demography reveals that seed and seedling transitions limit population persistence in a translocated shrub. Annals of Botany.

Harper JL (1977). Population biology of plants, Academic Press, London. 
Jongejans E, Shea K, Sheppard AW (2006). What controls the population dynamics of the invasive thistle Carduus nutans in its native range? J. Appl. Ecol., 43, 877-886.

Kalisz S, McPeek MA (1992). Demography of an age-structured annual: resampled projection matrices, elasticity analyses, and seed bank effects. Ecology, 73, 1082-1093.

Katzner TE, Ivy JAR, Bragin EA, Milner-Gulland E, DeWoody JA (2011). Conservation implications of inaccurate estimation of cryptic population size. Anim. Conserv., 14, 328332.

Kaye TN, Pyke DA (2003). The effect of stochastic technique on estimates of population viability from transition matrix models. Ecology, 84, 1464-1476.

Kisanuki H, Nakasu M, Nakai A, Yurugi Y (2008). Predicting the population dynamics of three understory broad-leaved evergreen species under the influence of Sika deer in primary and secondary forests of mid-western Japan. J. For. Res., 13, 52-58.

Kouassi KI, Barot S, Gignoux J, Bi IAZ (2008). Demography and life history of two rattan species, Eremospatha macrocarpa and Laccosperma secundiflorum, in Cote d'Ivoire. J. Trop. Ecol., 24, 493-503.

Logofet DO, Kazantseva ES, Belova IN, Onipchenko VG (2019). Disappointing viability forecat for a local population of Androsace albana in a random environment. Zh. Obshch. Biol., 80, 1-13.

Mondragon D (2009). Population viability analysis for Guarianthe aurantiaca, an ornamental epiphytic orchid harvested in Southeast Mexico. Plant Species Biol., 24, 35-41.

Morris WF, Doak DF (2002). Quantitative conservation biology: theory and practice of population viability analysis, Sinauer Associates, Sunderland, Massachusetts.

Parker IM (2000). Invasion dynamics of Cytisus scoparius: a matrix model approach. Ecol. Appl., 10, 726-743.

Quitete Portela RDC, Bruna EM, Maes dos Santos FA (2010). Demography of palm species in Brazil's Atlantic forest: a comparison of harvested and unharvested species using matrix models. Biodivers. Conserv., 19, 2389-2403.

R Core Team. 2015. R: A language and environment for statistical computing [Online]. Vienna, Austria: R Foundation for Statistical Computing. [Accessed 3rd October 2018].

Regan TJ, Chades I, Possingham HP (2011). Optimally managing under imperfect detection: a method for plant invasions. J. Appl. Ecol., 48, 76-85.

Salguero-Gómez R, Jones OR, Archer CR, Buckley YM, Che-Castaldo J, Caswell H, Hodgson D, Scheuerlein A, Conde DA, Brinks E, de Buhr H, Farack C, Gottschalk F, Hartmann A, Henning A, Hoppe G, Römer G, Runge J, Ruoff T, Wille J, Zeh S, Davison R, Vieregg D, Baudisch A, Altwegg R, Colchero F, Dong M, de Kroon H, Lebreton J-D, Metcalf CJE, Neel MM, Parker IM, Takada T, Valverde T, Vélez-Espino LA, Wardle GM, Franco M, Vaupel JW (2015). The COMPADRE Plant Matrix Database: an open online repository for plant demography. J. Ecol., 103, 202-218.

Salguero-Gómez R, Plotkin JB (2010). Matrix dimensions bias demographic inferences: implications for comparative plant demography. Am. Nat., 176, 710-722.

Shefferson RP (2009). The evolutionary ecology of vegetative dormancy in mature herbaceous perennial plants. J. Ecol., 97, 1000-1009.

Sletvold N, Rydgren K (2007). Population dynamics in Digitalis purpurea: the interaction of disturbance and seed bank dynamics. J. Ecol., 95, 1346-1359.

Stöcklin J, Fischer M (1999). Plants with longer-lived seeds have lower local extinction rates in grassland remnants 1950-1985. Oecologia, 120, 539-543. 
Tanner JE (2001). The influence of clonality on demography: patterns in expected longevity and survivorship. Ecology, 82, 1971-1981.

Venable DL (2007). Bet hedging in a guild of desert annuals. Ecology, 88, 1086-1090.

Verhulst J, Montana C, Mandujano MC, Franco M (2008). Demographic mechanisms in the coexistence of two closely related perennials in a fluctuating environment. Oecologia (Berl.), 156, 95-105.

Vitalis R, Glémin S, Olivieri I (2004). When genes go to sleep: the population genetic consequences of seed dormancy and monocarpic perenniality. The American Naturalist, 163, 295-311.

Warner RR, Chesson PL (1985). Coexistence mediated by recruitment fluctuations: a field guide to the storage effect. Am. Nat., 125, 769-787. 
Tables

Table 1. Comparison of studies from the MPM2008-2011 database with a previous review by

(Doak et al., 2002) examining the inclusion of the seed bank in plant population models. Studies were classified as justified exclusion if a valid reason was provided for not including the seed bank (e.g. the authors found no evidence of a seed bank) and unjustified exclusion if the possibility of a seed bank was not explored or mentioned. Studies from COMPADRE were not analysed to determine whether the exclusion of the seed bank was justified.

\begin{tabular}{lccc}
\hline $\begin{array}{l}\text { Population models and seed } \\
\text { banks }\end{array}$ & $\begin{array}{c}\text { Doak et al. } \\
(\mathbf{2 0 0 2})\end{array}$ & $\begin{array}{c}\text { MPM2008- } \\
\mathbf{2 0 1 1}\end{array}$ & $\begin{array}{c}\text { COMPADRE } \\
\mathbf{v . 5 . 0 . 0}\end{array}$ \\
\hline Included & $34(48.6 \%)$ & $16(29.1 \%)$ & $292(34.0 \%)$ \\
Justified exclusion & $6(8.6 \%)$ & $13(23.6 \%)$ & $568(66.0 \%)$ \\
Unjustified exclusion & $30(42.9 \%)$ & $26(47.3 \%)$ & \\
\hline Total studies & 70 & 55 & 860 \\
\hline
\end{tabular}


Table 2. Comparison of population growth rates $\left(\lambda_{1}\right)$ following the addition of a seed bank. Pop. refers to populations of the same species in the same study. Avg. represents the growth rate from the mean matrix for each population. Stoch. represents the mean stochastic growth rate from populations projected over 10,000 times. SB denotes single point estimates from models with germination and viability equal to that obtained from the literature. Growth rates from Monte Carlo simulations were obtained from the mean of 10,000 trials with parameters for germination, viability, seed survival, and seedling survival drawn from uniform distributions when priors are uninformed, while germination and viability were drawn from beta distributions when the priors were informed. $95 \%$ confidence intervals for Monte Carlo simulations and stochastic growth rates are displayed in round brackets.

\begin{tabular}{|c|c|c|c|c|c|c|c|}
\hline Study & $\begin{array}{c}\text { Life } \\
\text { Form }\end{array}$ & Pop & Period & No SB & SB & $\begin{array}{l}\text { Monte Carlo sim. } \\
\text { uninformed prior }\end{array}$ & $\begin{array}{l}\text { Monte Carlo sim. } \\
\text { informed prior }\end{array}$ \\
\hline \multicolumn{8}{|l|}{ Single time period } \\
\hline \multicolumn{8}{|l|}{ Chien et al. (2008) } \\
\hline Calocedrus macrolepus & Tree & 1 & 1 & 0.9683 & 0.9715 & $0.9700(0.9683,0.9785)$ & $0.9699(0.9683,0.9752)$ \\
\hline Parashorea chinensis & Tree & 1 & 1 & 0.9948 & 0.9955 & $0.9963(0.9948,1.0040)$ & $0.9954(0.9948,0.9978)$ \\
\hline Pinus kwangtungensis & Tree & 1 & 1 & 0.9776 & 0.9786 & $0.9786(0.9776,0.9834)$ & $0.9782(0.9776,0.9799)$ \\
\hline \multicolumn{8}{|l|}{ Kisanuki et al. (2008) } \\
\hline \multirow[t]{2}{*}{ Illicium anisatum } & Tree & 1 & 1 & 1.1271 & 1.1647 & $1.1344(1.1258,1.1765)$ & $1.1408(1.1263,1.1778)$ \\
\hline & & 2 & 1 & 1.1864 & 1.2276 & $1.1931(1.1828,1.2363)$ & $1.2008(1.1840,1.2416)$ \\
\hline \multicolumn{8}{|l|}{ Abe et al. (2008) } \\
\hline \multirow[t]{3}{*}{ Sambucus racemosa } & Shrub & 1 & 1 & 1.1696 & 1.1690 & $1.1789(1.1661,1.2355)$ & $1.1951(1.1674,1.2639)$ \\
\hline & & 2 & 1 & 1.0007 & 1.0012 & $1.0109(1.0007,1.0622)$ & $1.0255(1.0008,1.0963)$ \\
\hline & & 3 & 1 & 2.2227 & 2.2031 & $2.1588(2.0468,2.2210)$ & $2.1459(2.0551,2.2125)$ \\
\hline \multicolumn{8}{|l|}{ Kouassi et al. (2008) } \\
\hline Eremospatha macrocarpa & Tree & 1 & 1 & 0.9792 & 0.9811 & $0.9806(0.9792,0.9887)$ & $0.9805(0.9792,0.9842)$ \\
\hline
\end{tabular}




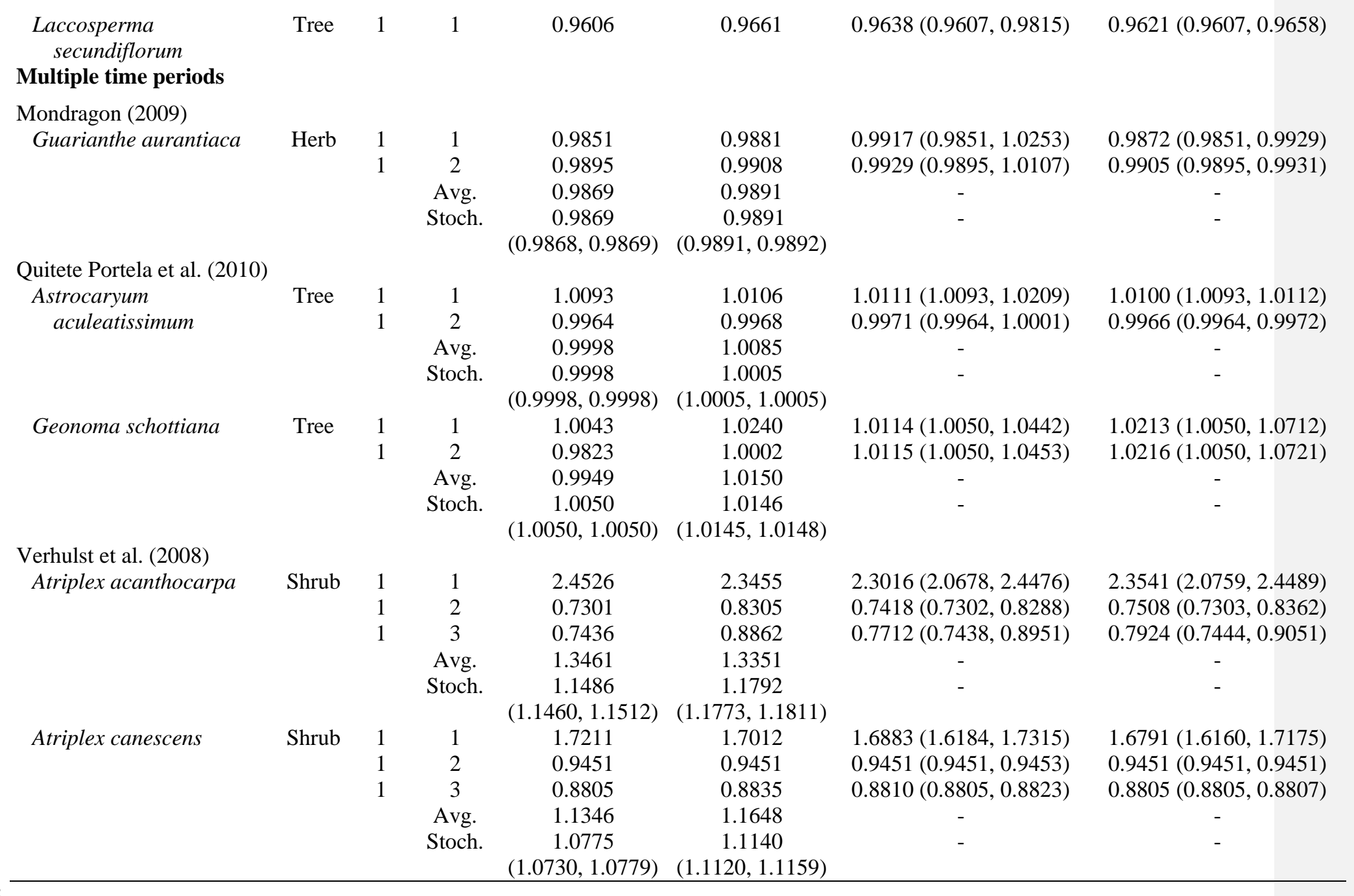


604 Table 3. Comparison of population growth rates following removal of the seed bank. Studies are classified on the basis of variability 605 in transition elements that was reported or number of time periods that were provided. Pop. refers to different populations of the same 606 species in the same study. Avg. represents the growth rate from the mean matrix. Stoch. represents the mean stochastic growth rate 607 from populations projected over 10,000 times. Confidence intervals for stochastic simulations when the seed bank was removed are 608 shown in brackets.

\begin{tabular}{|c|c|c|c|c|c|}
\hline Study & Life Form & Pop. & Period & SB & No SB \\
\hline \multicolumn{6}{|l|}{ No variability reported } \\
\hline $\begin{array}{l}\text { Adams et al. (2005) } \\
\text { Helenium virginicum } \\
\text { de Kroon et al. (1987) }\end{array}$ & Herb & 1 & 1 & 1.1145 & 0.6985 \\
\hline Hypochaeris radicata & Herb & 1 & 1 & 0.2973 & 0.2154 \\
\hline \multicolumn{6}{|c|}{ Variability, single time period } \\
\hline $\begin{array}{l}\text { Jongejans et al. (2006) } \\
\text { Carduus nutans }\end{array}$ & Herb & $\begin{array}{l}1 \\
2 \\
3\end{array}$ & $\begin{array}{l}1 \\
1 \\
1\end{array}$ & $\begin{array}{l}0.4680 \\
0.6270 \\
0.6020\end{array}$ & $\begin{array}{l}0.3894 \\
0.3771 \\
0.5524\end{array}$ \\
\hline \multicolumn{6}{|l|}{ Multiple time periods } \\
\hline $\begin{array}{l}\text { Angert (2006) } \\
\text { Mimulus lewisii }\end{array}$ & Herb & $\begin{array}{l}1 \\
1 \\
1 \\
1 \\
1 \\
\\
2 \\
2\end{array}$ & $\begin{array}{c}1 \\
2 \\
3 \\
\text { Avg. } \\
\text { Stoch. } \\
\\
1 \\
2\end{array}$ & $\begin{array}{c}0.7778 \\
0.6863 \\
0.9959 \\
0.8541 \\
0.8423 \\
(0.8410,0.8435) \\
1.0154 \\
0.6978\end{array}$ & $\begin{array}{c}0.7778 \\
0.6815 \\
0.9943 \\
0.8332 \\
0.8181 \\
(0.8222,0.8233) \\
1.0053 \\
0.6917\end{array}$ \\
\hline
\end{tabular}




\section{Parker (2000)}

Cytisus scoparius

\begin{tabular}{|c|c|c|c|}
\hline 2 & 3 & 1.3203 & 1.3746 \\
\hline 2 & Avg. & 1.0158 & 1.0410 \\
\hline \multirow[t]{2}{*}{2} & Stoch. & 0.9642 & 0.9949 \\
\hline & & $(0.9769,0.9787$ & $(0.9935,0.9963)$ \\
\hline 3 & 1 & 1.1052 & 1.1094 \\
\hline 3 & 2 & 1.1197 & 1.1450 \\
\hline 3 & 3 & 0.9523 & 0.9507 \\
\hline 3 & Avg. & 1.0510 & 1.0583 \\
\hline \multirow[t]{2}{*}{3} & Stoch. & 1.0472 & 1.0537 \\
\hline & & $(1.0470,1.0500)$ & $(1.0544,1.0557)$ \\
\hline 4 & 1 & 1.5841 & 1.6704 \\
\hline 4 & 2 & 1.0459 & 1.0470 \\
\hline 4 & 3 & 1.1227 & 1.3083 \\
\hline 4 & Avg. & 1.3112 & 1.3448 \\
\hline \multirow[t]{2}{*}{4} & Stoch. & 1.2919 & 1.3198 \\
\hline & & $(1.2908,1.2930)$ & $(1.3192,1.3205)$ \\
\hline 1 & 1 & 2.1963 & 2.3331 \\
\hline 1 & 2 & 2.2629 & 2.3858 \\
\hline 1 & Avg. & 2.5019 & 2.7128 \\
\hline \multirow[t]{2}{*}{1} & Stoch. & 2.4919 & 2.6927 \\
\hline & & $(2.4879,2.4960)$ & $(2.6868,2.6986)$ \\
\hline 2 & 1 & 1.7097 & 1.7407 \\
\hline 2 & 2 & 1.2542 & 1.2406 \\
\hline 2 & Avg. & 1.5681 & 1.5552 \\
\hline \multirow[t]{2}{*}{2} & Stoch. & 1.5507 & 1.5351 \\
\hline & & $(1.5495,1.5518)$ & $(1.5343,1.5359)$ \\
\hline 3 & 1 & 1.0040 & 1.0040 \\
\hline 3 & 2 & 0.9295 & 0.9287 \\
\hline 3 & Avg. & 0.9748 & 0.9746 \\
\hline \multirow[t]{2}{*}{3} & Stoch. & 0.9740 & 0.9738 \\
\hline & & $(0.9734,0.9746)$ & $(0.9700,0.9776)$ \\
\hline
\end{tabular}


Sletvold and Rydgren (2007)

Digitalis purpurea

\begin{tabular}{|c|c|c|c|c|}
\hline \multirow[t]{10}{*}{ Herb } & 1 & 1 & 0.7533 & 0.3174 \\
\hline & 1 & 2 & 0.7831 & 0.5536 \\
\hline & 1 & Avg. & 0.7715 & 0.5071 \\
\hline & 1 & Stoch. & 0.7713 & 0.5008 \\
\hline & & & $(0.7712,0.7713)$ & $(0.5002,0.5013)$ \\
\hline & 2 & 1 & 0.6831 & 0.2983 \\
\hline & 2 & 2 & 1.0198 & 0.9379 \\
\hline & 2 & Avg. & 0.8565 & 0.6701 \\
\hline & 2 & Stoch. & 0.8388 & 0.5794 \\
\hline & & & $(0.8384,0.8392)$ & $(0.5787,0.5802)$ \\
\hline
\end{tabular}




\section{Figures}

610 Fig. 1. Life cycle graphs corresponding to: A) a plant life cycle consisting of two stages: the seedlings, Se, and the adults, Ad; B) a plant life cycle consisting of seeds that have remained in the seed bank for 1 year, S1, up to $n$ years, Sn (Eq. 1), and C) a plant life cycle with an unstructured seed bank, SB, (Eq. 2). The transition rate $f_{\mathrm{Se}, \mathrm{Ad}}$ gives the fecundity into the seedling stage while $f_{\mathrm{S} 1 \text {,Ad }}$ and $f_{\mathrm{SB}, \mathrm{Ad}}$ gives the fecundity into the seed bank. D) Details of the derivation of Eq. 1 in which the parameters are contained in boxes (see formal notation in the text). Tracking the vital rates required to reach the seedling stage from the adult stage either directly or via the seed bank demonstrates how each term in Eq. 1 was derived.

Fig. 2. Scatterplot comparing population growth rates between models when the seed bank is included (y-axis) and when the seed bank is not included (x-axis). "Addition" refers to models in which we added the seed bank the model (Table 2), while "Removal" refers to models where we removed the seed bank (Table 3). Growth rates for models where the seed bank was added were taken from Monte Carlo simulations with germination prior guided by the literature. Points above the one-to-one line indicate the seed bank has a positive effect on $\lambda$ while points below indicate a negative effect. Points within the red section are declining populations $\left(\lambda_{1}<1\right)$ while those outside are increasing populations $\left(\lambda_{1}>1\right)$. acanthocarpa from A) 1996-1997, B) 1997-1998, and C) 1998-1999 based on 10,000 Monte Carlo simulations calculated using an uninformed prior on germination (uniform distribution) and an informed prior on germination (beta distribution with the mode equal to the germination 
rate obtained from the literature). Red dashed lines indicate the mean growth rate using uninformed and informed priors as reported in Table 2.

Fig. 4. Population growth rate $\left(\lambda_{1}\right)$ as a function of germination rate from the seed bank for $\left.\mathbf{A}\right)$ Atriplex acanthocarpa, B) Atriplex canescens, C) Illicium anisatum, and D) Geonoma schottiana. Population growth rates were calculated at seed viability rates of 1 (green line), 0.5 (blue line), and 0.1 (red line) for the germination rates between 0 and 1 at intervals of 0.01 . The germination rate for G. schottiana is restricted between 0 and 0.69 to not exceed the total seed survival estimate obtained from the literature.

Fig. 5. Flow chart describing different approaches to dealing with a potential seed bank when modelling plant population dynamics. When the presence of the seed bank is uncertain, it is advised that researchers explore this possibility either through literature confirmation, or investigating the soil reserves for viable seeds. By not investigating, the exclusion is unjustified, resulting in the highest possible uncertainty. Justified exclusion, by either proving it does not exist or showing it is unimportant via simulations, gives the highest level of certainty in the model outcomes. Including the seed bank, either by using the literature or data obtained in the field would unavoidably result in some amount of uncertainty from estimating the vital rates, but is preferable to unjustified exclusion. 
651 Fig. 1

A

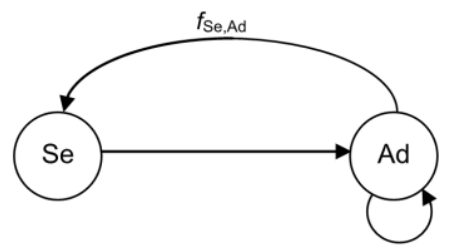

B

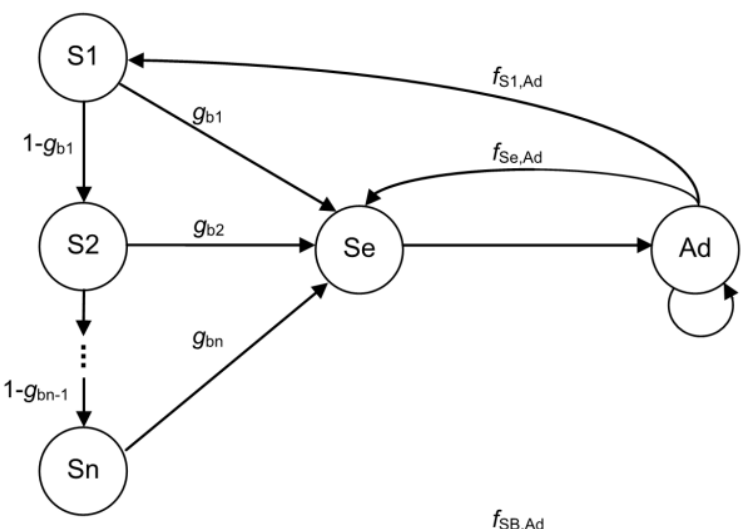

C

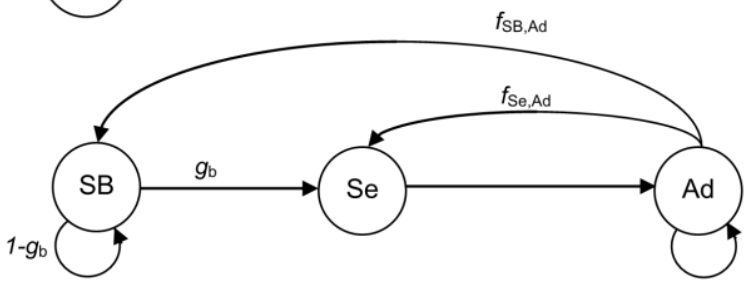

D

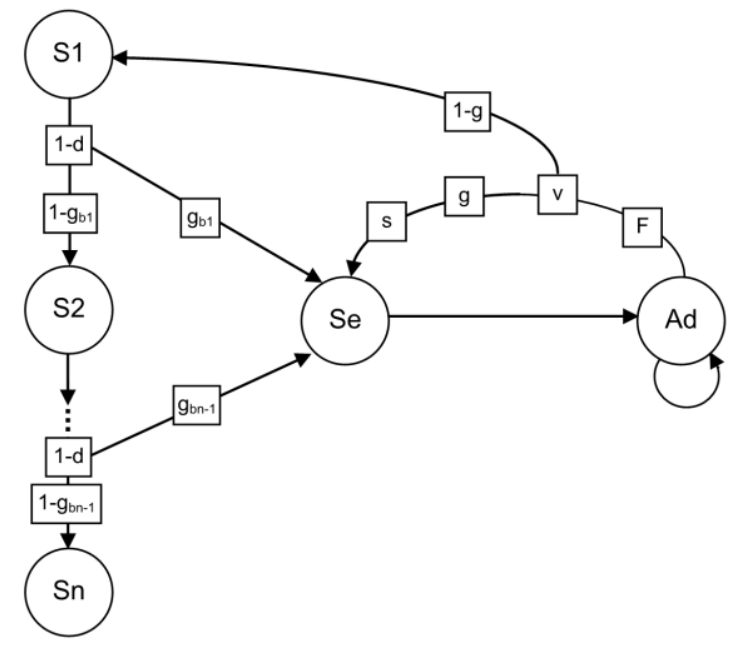


653 Fig. 2

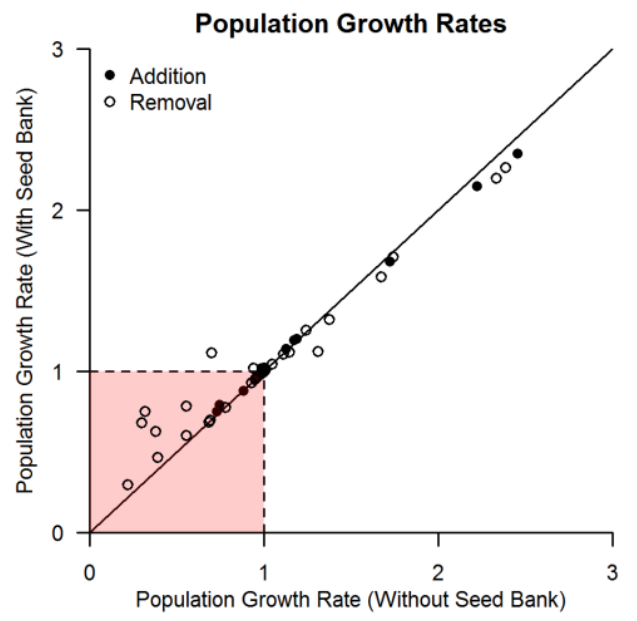


655 Fig. 3
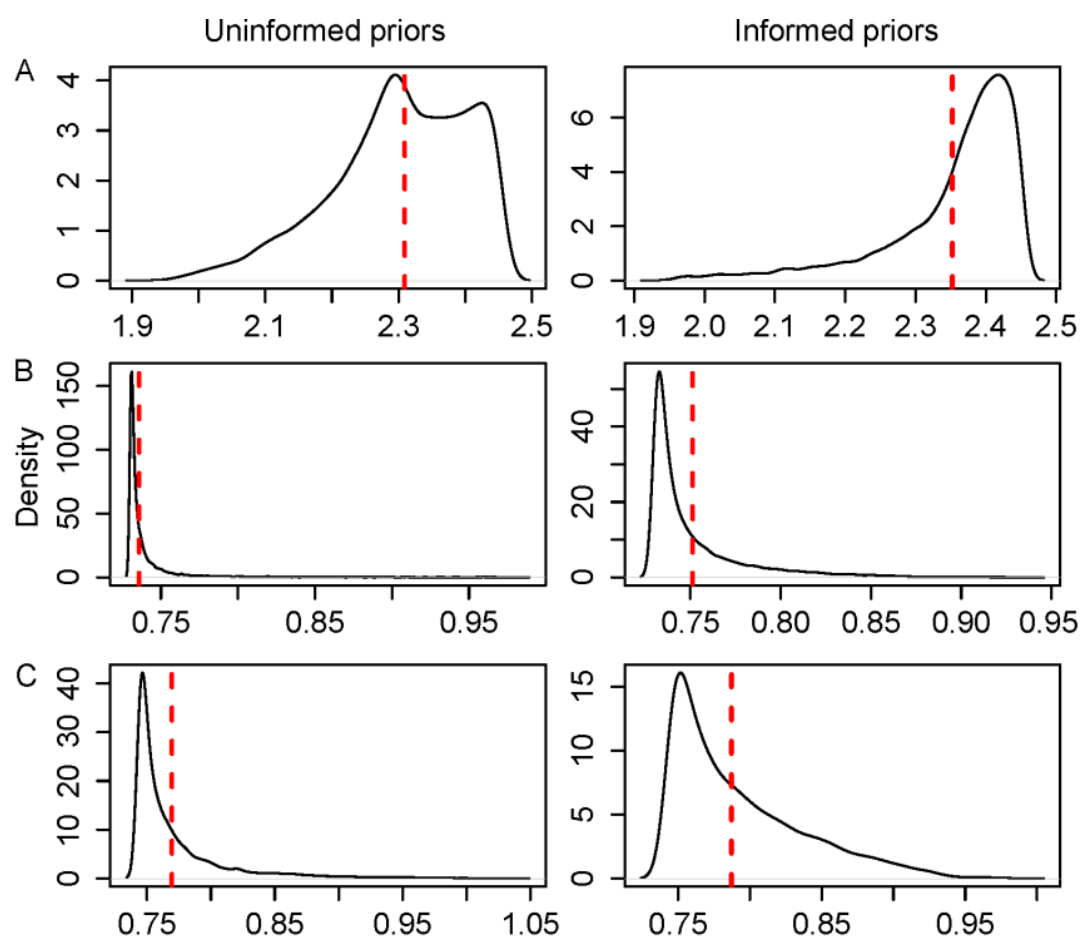

Population growth rate 
Fig. 4
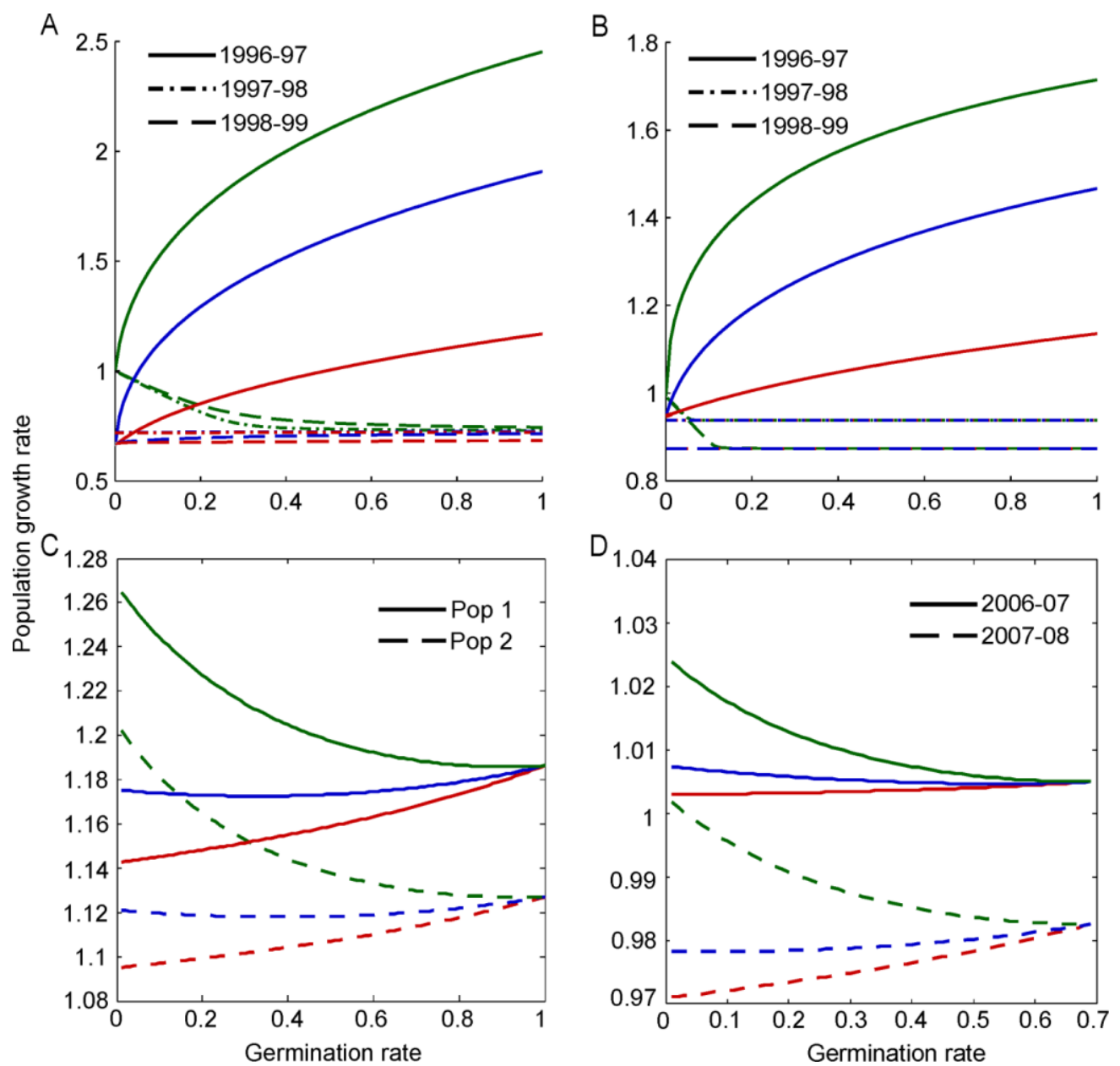
Fig. 5

\section{Source of uncertainty}

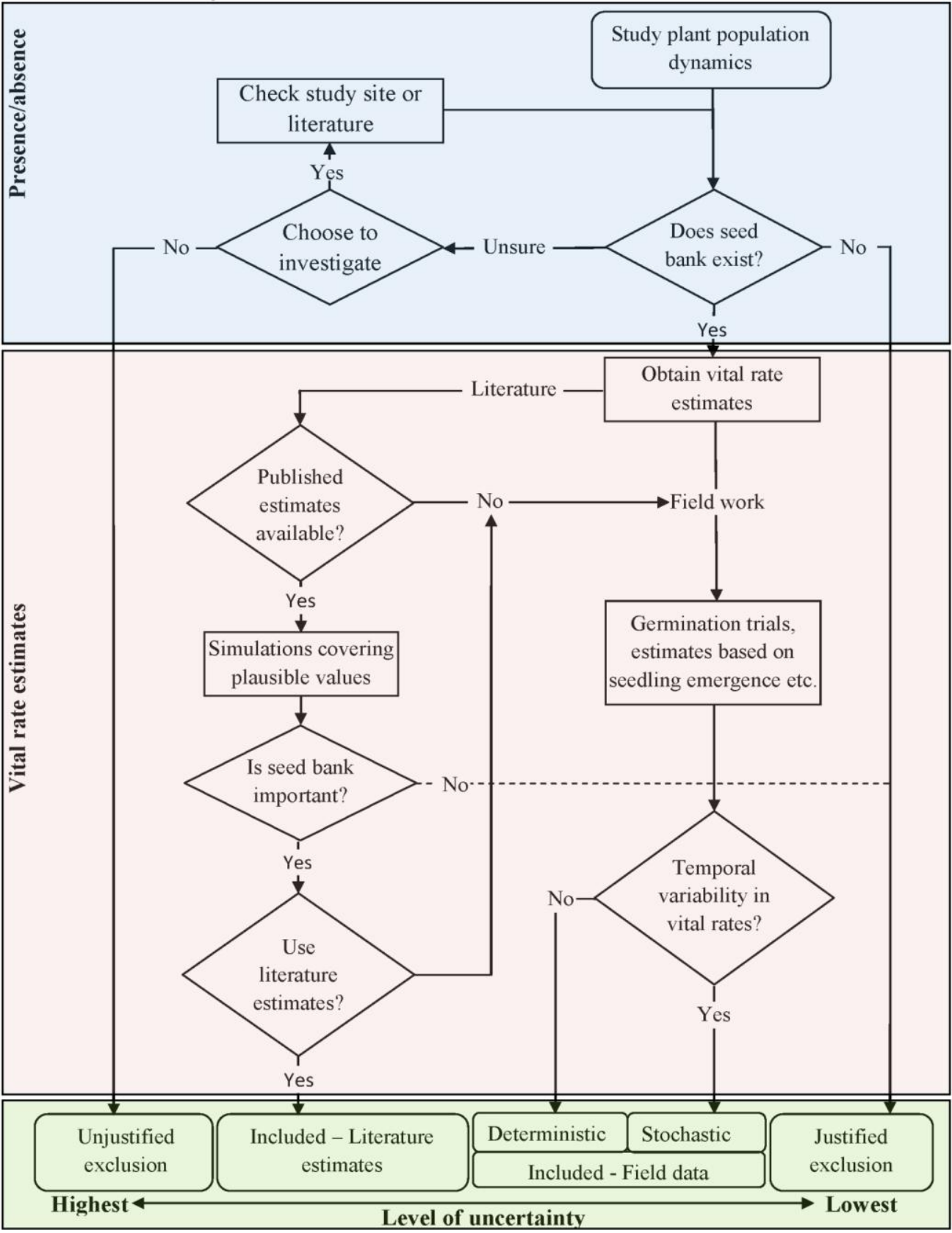




\section{Supporting Information}

\section{Table S1}

664 Population growth rates from seed bank models, Monte Carlo simulations and alternative

665 formulations with 2 and 3 year longevity

666 Table S2

667 Elasticity matrices for 1997 and 1998 for Atriplex acanthocarpa with and without the seed bank

\section{Figure S1}

669 Log population size projections for single point matrices with and without the seed bank

\section{$670 \quad$ Figure S2}

671 Cumulative extinction probabilities of quasi-extinction

\section{Method S1}

673 Example demonstrating methodology for including the seed bank stage and alternative

674 formulations

\section{Method S2}

676 Example of seed bank stage removal from a matrix model for Digitalis purpurea

\section{$677 \quad$ File S1}

678 Excel file containing original and updated matrices used for addition and removal simulations 
Highlights (for review)

- Inclusion of seed bank reduced extinction times and improved long-term persistence

- Published literature and simulations can assess the importance of the seed bank

- Inclusion of the seed bank may change model predictions made otherwise 
1

2

3

\section{Consequences of neglecting cryptic life stages from demographic models}

2

3 Vuong Nguyen ${ }^{1}$, Yvonne M. Buckley ${ }^{2,3}$, Roberto Salguero-Gómez ${ }^{2,4,5}$ and Glenda M. Wardle ${ }^{1}$ 4

$5{ }^{1}$ Desert Ecology Research Group, School of Life and Environmental Sciences, The University of

6 Sydney, Sydney, NSW 2006, Australia

$7 \quad{ }^{2}$ School of Biological Sciences, The University of Queensland, Queensland, 4072, Australia

$8 \quad{ }^{3}$ School of Natural Sciences, Department of Zoology, Trinity College Dublin, Dublin, Ireland

$9{ }^{4}$ Evolutionary Biodemography Laboratory, Max Planck Institute for Demographic Research,

10 Rostock 18057, Germany

$11{ }^{5}$ Department of Zoology, University of Oxford, Oxford, UK

12

13 *Correspondence author. E-mail: phuc.nguyen@sydney.edu.au

14 
16 Abstract

17 Information on individuals from all stages of life is crucial to explore their ecology, evolution

18 and conservation biology. However, the life cycles of many species contain cryptic life stages

19 that are difficult to detect and track over time and are therefore omitted from demographic

20 models. One example is the dormant seed bank, an evolutionary bet-hedging mechanism that

21 buffers plant populations in variable environments. To evaluate this methodological oversight,

22 we conduct simulations to explore the effect of seed bank parameter uncertainties on

23 demographic outputs such as the deterministic $\left(\lambda_{1}\right)$ and stochastic population growth rate $\left(\lambda_{\mathrm{S}}\right)$,

24 and extinction probabilities of 12 plant species. We have used uninformed and informed priors

25 for seed bank parameters based on literature estimates, and reconstructed published models in

26 which the seed bank was excluded without justification. Trials on removing the seed stage from

27 models (6 species) explored the worst-case scenario for ignoring the seed bank. Inclusion of a

28 seed bank and demographic uncertainty in seed bank parameters have little impact on stable

29 populations $\left(\lambda_{1} \approx 1\right)$ with high post-seedling survival. When populations deviate from stability or

30 demonstrate temporal demographic variation, greater changes in $\lambda_{1}$ and the range of possible

31 growth rates caused by demographic uncertainty are observed. As expected, decreasing

32 populations $\left(\lambda_{1}<1\right)$ benefit from the inclusion of a seed bank through increases in the growth

33 rate and extinction times, whereas increasing populations $\left(\lambda_{1}>1\right)$ are slowed down. While

34 germination estimates from the literature cannot accurately reflect those obtained in the field,

35 they provide a starting point to assess the relative importance of a seed bank. The exclusion of

36 the seed bank must be justified by confirming that dormancy is either non-existent or not

37 important. Accounting for cryptic stages in demographic models will produce better informed

38 management decisions for threatened or invasive species. 
39 Keywords: Comparative demography, cryptic life stages, matrix population models, plant

40 population ecology, seed bank, seed dormancy.

41

42 Highlights

43 - Inclusion of seed bank reduced extinction times and improved long-term persistence

44 - Published literature and simulations can assess the importance of the seed bank

45 - Inclusion of the seed bank may change model predictions made otherwise 


\section{Introduction}

49 Demographers have long recognised that individuals in a population span across different stages

50 of their life cycle, from propagules up to reproductive adults, and that these stages contribute to

51 its persistence in different ways - failing to account for all these stages can therefore be

52 misleading (Caswell, 2001). However, the life cycles of many species contain cryptic life stages

53 that may be difficult to detect or sample. These cryptic life stages occur over a range of

54 taxonomic groups, and may include hibernating or dormant individuals, clonal individuals, eggs,

55 mobile propagules, or simply stages occupying inaccessible locations (Geiser, 2004, Katzner et

56 al., 2011, Shefferson, 2009, Tanner, 2001). The dormant seed bank is perhaps one of the most

57 common examples of the cryptic life stage whereby seeds enter prolonged dormancy (>1 year)

58 within the soil or above ground in woody cones, and can make up a significant portion of the

59 population (Baskin and Baskin, 2014, Harper, 1977). By spreading germination through time,

60 seed dormancy acts as a bet-hedging mechanism mitigating the effects of unfavourable years in

61 exchange for reduced proliferation during favourable years (Gremer et al., 2012, Venable, 2007).

62 In extreme cases, seed banks can prevent local extinctions when above-ground populations are

63 killed off entirely (Stöcklin and Fischer, 1999). Seed dormancy has also been shown to promote

64 species co-existence in variable environments (Warner and Chesson, 1985) and act as a temporal

65 reservoir for genetic diversity (Vitalis et al., 2004).

66 With such substantial consequences for population dynamics, it would be a major

67 oversight to exclude the seed bank, or any cryptic life stage, from population estimates and

68 demographic analyses. This argument is indeed not new: the need to incorporate the seed bank

69 into demographic models has been recognised for decades (Harper, 1977, Kalisz and McPeek,

70 1992). Matrix population models, which divide individuals into their respective stage classes, are 
71 the most common tool for modelling plant populations - the online database of plant matrix

72 models, COMPADRE v5.0.0 contains 8,906 published matrices for 759 species ( COMPADRE

73 Plant Matrix Database, v5.0.0., 2019, Salguero-Gómez et al., 2015). These models have a long

74 history in the management of threatened and invasive species (Crone et al., 2011). Yet, a

75 previous review of 70 published matrix population models found almost half (43\%) had

76 excluded the seed bank stage without justification (Doak et al., 2002). Surprisingly, we have

77 found that this proportion has remained unchanged in the last decade (47\%; Table 1). We

78 acknowledge that including the seed bank is not straightforward as the estimation of vital rates

79 such as survival and germination generally rely on additional laboratory experiments (Crone et

80 al., 2011), and even these would inflate actual in situ estimates (Gross and Mackay, 2014).

81 Furthermore, unless such experiments are rigorous enough to include age-specific vital rates

82 (Kalisz and McPeek, 1992), the seed bank has been generally assumed to be unstructured.

83 Excluding the seed bank may be justified if it is discovered the species, or population, does not

84 possess dormant seeds (see Baskin and Baskin, 2014 for review on the presence of seed

85 dormancy), or if it is mathematically and empirically justified in a modified model (Logofet et al.

86 2017), however, these cases are the minority compared to those that do not provide justification

87 (Doak et al., 2002; Table 1). Unjustified exclusion can have real-world consequences if, for

88 example, the seed bank of an invasive species recolonises after the adult individuals have been

89 eliminated (Regan et al., 2011), or if the population size and persistence of threatened species is

90 underestimated (Doak et al., 2002).

91 The practical issues of including the seed bank and its exclusion from demographic

92 models raise two important questions. First, how can we bridge the gap in our understanding of

93 whether a seed bank is necessary to adequately model the population dynamics in the absence of 
94 field data? Second, how does uncertainty in the presence of a seed stage and its transition rates

95 translate into uncertainty in the model? Addressing these questions using a simulation-based

96 approach can be useful for investigating a range of scenarios and assumptions but may not be

97 biologically meaningful without application to real-world examples. An empirical approach, on

98 the other hand, may be limited in its scope if it were to focus on a single case study, as there are

99 clear difficulties in obtaining individual-level records for published models. Using published

100 matrix models that have excluded the seed bank without justification and information on

101 dormancy and germination rates from the literature, we implement a combination of these

102 approaches and perform a comparative analysis across a range of species and life forms (12

103 species including eight trees, three shrubs and one herb). We conduct Bayesian Monte Carlo

104 simulations on these published models using uninformed and informed priors (based on literature

105 estimates) for seed bank vital rates to gauge the level of uncertainty when the seed bank presence

106 and parameters are unknown. To complement these addition simulations, we also investigate the

107 implications of assuming the absence of a persistent seed bank by intentionally removing the

108 seed bank from models which did include it.

109 We make a number of predictions based on the evolutionary advantages of seed

110 dormancy. First, we might expect the relative importance of seed banks to be greater for species

111 in unpredictable environments with variable post-seedling survival compared to stable

112 populations of long-lived species with consistently high adult survival (Venable, 2007).

113 Furthermore, the deterministic growth rate $\left(\lambda_{1}\right)$, which assumes vital rates remain constant,

114 should increase during poor demographic years $\left(\lambda_{1}<1\right)$ and decrease during productive years $\left(\lambda_{1}\right.$

$115>1)$ to reflect the function of the seed bank as a bet hedging mechanism against unfavourable years (Gremer et al., 2012, Kalisz and McPeek, 1992). Similarly, elasticities (the relative 
117 influence of each demographic process to the population growth rate; de Kroon et al., 2000) for 118 seed bank transition rates should be greater during poor years. We should also observe increases 119 in times to local extinction and stochastic growth rates $\left(\lambda_{\mathrm{S}}\right)$ which incorporate temporal variation 120 in vital rates. Using both an empirical and simulation-based approach, we provide more robust 121 projections of the demographic fates of populations and help to motivate the inclusion of the 122 seed bank into demographic models.

\section{Materials and Methods}

\section{Database of population matrix models}

125 At the time this study was conducted, the COMPADRE Plant Matrix Database had not yet 126 become open-access online (Salguero-Gómez et al., 2015), with the most recent update 127 consisting of 204 terrestrial plant species up to 2007, which we hereafter refer to as the 128 "MPM2007" database constructed by the Vegetation Function Working Group (see Burns et al., 1292010 for further information). We have used the ISI Web of Science to identify published plant 130 matrix population models from 2008-2011, which we hereafter refer to as the "MPM2008-2011" 131 database. These studies would later go on to be added to the COMPADRE database. Examples 132 of keywords used in our search include "matrix model*", "transition matrix", "demographic 133 model*", "population model*”, "population viability analysis", "population growth rate”, and 134 "population dynamics". The addition of * at the end of a search term acts as a wildcard and 135 allows for alternative forms of a given word, e.g. model(s), model(ling). We have found 55 136 studies with matrix models for 77 terrestrial plant species including 38 trees, 27 herbs, 8 shrubs 137 and 4 succulents.

138 Confirmation of the seed bank 
Using Baskin and Baskin (2014) and the species name as a search term in ISI Web of Science, we have verified whether a seed bank was reported for species in which seed bank exclusion was

141 unjustified (by not mentioning the seed bank; Table 1). While each population may not

\section{Addition and removal of seed bank stages}

Adding, removing, or excluding stages without justification alters the dimensionality of the model and this will affect the demographic parameter estimates. Merging or further dividing up stage classes directly alters vital rates for each class being affected and the speed of the life cycle, where decreasing the dimensionality reduces the number of stages to progress through the 

162 bet hedging mechanism, delayed movement through the life cycle is to be expected and this is 163 reflected in our hypotheses. Since the seed bank is a separate, discrete stage class that involves 164 no splitting or merging, transition rates for the other classes should remain unaffected by its 165 inclusion. However, seedling fecundity would need to be lowered accordingly to account for observed seedlings emerging from the seed bank to avoid overestimating fecundity. We use seedling counts to estimate fecundity in all models where the seed bank was excluded without justification. For simplicity, we assume no clonal reproduction into the seedling stage such that the observed seedlings only consist of two components: the individuals that germinated immediately between year $t$ and $t+1$ and those that germinated from the dormant seed bank from prior years. The number of observed seedlings can thus be described by the following equation:

$$
f_{i, j}=F_{j}(t) v g s+F_{j}(t-1) v(1-g)(1-d) g_{b 1}+\mathrm{F}_{j}(t-2) \mathrm{v}(1-g)\left(1-g_{b 1}\right)(1-d)^{2} g_{b 2}+\ldots
$$

where the first term on the right hand side represents immediate germinants, the second term represents germination of seeds from the seed bank produced at the previous time step, the third term represents germination of seeds from the seed bank produced two time steps ago, and so on. Notations:

$f_{i, j}=$ per-capita fecundity into stage class $i$ by stage class $j$ in one time step; $F_{j}(t)=$ total fecundity by stage class $j$ at time $t$; $v=$ seed viability rate during the step $t$ to $t+1$; $g=$ non-seed bank germination rate at the period $t$ to $t+1$; $g_{b l}=$ germination rate from seeds that remained dormant in the seed bank for 1 time step;

life cycle and vice versa (Salguero-Gómez and Plotkin, 2010). As the seed bank functions as a 
$g_{b 2}=$ germination rate from seeds that remained dormant in the seed bank for 2 time steps;

$s=$ seedling survival rate until the next census;

$d=$ seed bank mortality rate

187 See Fig. 1 for more information.

188 To simplify the inclusion of the seed bank stage under limited information, we have made the

189 following assumptions. First, the seedling survival rate $(s)$ from the period $t$ to $t+1$, is equal to

190 the seed bank survival rate $(1-d)$. Second, we start with simulations that focus on the

191 contribution of the seed bank from $t-1$. This unstructured submodel captures the predominant

192 seed bank contribution since the contribution of seeds from previous years will diminish

193 exponentially with each year if we assume the vital rates to be not seed age dependent.

194 Alternative age-structured formulations are explored below. Additionally, we assume the

195 fecundity from the previous year to equal the fecundity in the current year, i.e. $F_{j}(t)=F_{j}(t-1)$,

196 hereafter denoted by $F_{j}$. Finally, the probability of germinating within the census year is equal to

197 the probability of germinating from the seed bank, i.e. $g=g_{b}$ (Kalisz and McPeek, 1992). Using

198 the published information on germination, we simplify Eq. 1:

$$
f_{i, j}=F_{j} v g s+F_{j} v(1-g)(1-d) g
$$

where the first term on the right-hand side represents the number of seedlings germinating immediately between $t$ and $t+1$, and the second term corresponds to germination from the seed bank. For estimates of the deterministic and stochastic growth rates, we set seed viability $(v)$, seedling survival $(s)$ and seed survival $(1-d)$ equal to 1 unless this information was provided in the literature alongside germination estimates. Monte Carlo simulations (described below) then 
investigate drawing these values from both uninformed and informed prior distributions. Taking the observed $f_{i, j}$ from the original matrix, we solve Eq. 2 for $F_{j}$ and calculate the per-capita fertility contributions to seedlings and seed bank (See Supplementary Method S1 for an example). Following the same approach, we also explore alternative model formulations and the effect of different assumptions but only perform Monte Carlo simulations for the unstructured scenario, which is the simplest and most common approach to including the seed bank. The alternatives include the addition of an age-structured seed bank distinguishing seeds from $t-2$ and $t-3$ and more conservative estimates on the effect of the seed bank by further assuming the seeds do not survive beyond the first, second or third year (See Supplementary Method S1 and Table S1 for the alternative formulations and population growth rates for all models).

Researchers excluding the seed bank from a model make the implicit assumption that it is not present in the population. Thus we intentionally remove the seed bank from selected models to explore the implications of this assumption. Given the large number of potential models to choose from, we have decided to focus on cases in which the seed bank was known to be important (elasticities of $\lambda_{1}$ to seed survival, germination or fecundity $>0.1$ ) to demonstrate the worst case scenario that might be possible when the seed bank is ignored. We have identified six such species from the MPM2007 and MPM2008-2011 databases that fulfil this criteria whilst retaining strong connectedness, the condition that it is possible to transit from each node to all other nodes of the life cycle graph (Caswell, 2001), such as those in Figure 1. We use information on seed viability, seedling survival, and seed survival if provided, but otherwise we set them to 1 , and we no longer assume that $g=g_{b}$ since $g_{b}$ can be obtained from the original matrix. We estimate the rate of seed germination within the studied period, $g$, based on partitioning the total reproductive output (Eqs. 1 and 2) to recalculate only the fecundity into the 
seedling stage. To further ensure that no artefacts from changes to reproduction or mortality are introduced, the removal protocol is the reverse of the addition protocol, and we check that the can be recovered by reapplying the addition protocol (See Supplementary Method S2 for an example).

\section{Analyses}

We use R (v 3.1.1; The R Foundation for Statistical Computing) to calculate population growth rates $\left(\lambda_{1}\right)$, elasticities, stochastic simulations exploring temporal variation in vital rates and Monte Carlo simulations exploring uncertainty in seed bank vital rates (see Supplementary File S2 for code to perform Monte Carlo simulations).

We use uninformed priors for germination, viability, seedling survival and seed survival for Monte Carlo simulations by drawing these rates from a uniform distribution. Although we might expect covariance among vital rates for the below- and above-ground individuals (Morris and Doak, 2002), introducing this structure is complicated particularly when dealing with the seed bank. For example, models that do include the seed bank generally estimate seed bank vital rates through separate germination experiments such that the covariance between the seed bank and the above-ground population is lost in the process. While the impact of this covariance structure on the model outcomes would be of interest, we instead focus here on the exclusion of the seed bank from demographic models and leave investigations regarding correlation structure for future research.

We perform 10,000 simulations, recalculating the fecundities and reconstructing the matrix for each set of parameters, and report the mean growth rate with confidence intervals obtained by the $2.5 \%$ and $97.5 \%$ quantiles. We then determine the effect of using informed priors via 
literature estimates for germination, and occasionally viability when available, on the resulting probability density function (p.d.f.) of the distribution of growth rates. Publications on germination often contain multiple treatments and an estimated mean germination rate for each treatment. We have specified our prior distributions depending on the type of demographic year to reflect the function of the seed bank as a bet hedging mechanism (the highest germination rate available when $\lambda_{1}>1$ and the lowest when $\lambda_{1}<1$; Gremer et al., 2012, Kalisz and McPeek, 1992). However, if only one germination rate was provided, we use this value for all population matrix models regardless of the demographic year. If only the mean matrix was provided (arithmetic mean matrix across all years and sites), we use the average germination over all treatments. We model both germination and viability using the beta distribution as it varies between 0 and 1 , with the distribution mode equal to the germination rate. We also calculate $\lambda_{1}$ for fixed values of the germination rate $(0.01-1$ by increments of 0.01$)$ and viability rate $(0.1,0.5$, and 1) to capture the total range of possible values when only these parameters are allowed to vary. The matrices in which the germination rate was set to that obtained from the literature are used to calculate single point estimates of $\lambda_{1}$, elasticity matrices, $\lambda_{\mathrm{S}}$ and extinction probability, with viability, seed survival and seedling survival set to 1 if no information was available. The initial population for all stochastic and extinction probability calculations hereafter consists of 1,000 individuals at the stable stage distribution, calculated as the normalized dominant right eigenvector (Caswell, 2001) of the mean matrix. We have calculated stochastic growth rates via random choice from a set of available matrices (Kaye and Pyke, 2003), where each matrix has an equal probability of being selected at each time step. The initial population is projected over 271500,000 time steps with the first 5,000 omitted to remove transient dynamics (Kaye and Pyke, 
2003, Morris and Doak, 2002). We use the mean and variance of the growth rates to obtain an estimate for $\lambda_{\mathrm{S}}$ and $95 \%$ confidence intervals.

We also compare extinction probabilities between models with and without seed banks. Times to extinction (defined as population size $\leq 1$ individual) are calculated for matrices predicting population decline. We project an initial population for individual matrices over 100 years and compared the difference in times to extinction with and without the seed bank to quantify the effect of the seed bank on population persistence. We then conduct stochastic simulations to calculate the cumulative probability of quasi-extinction (Morris and Doak, 2002) with a quasi-extinction threshold set to 100 individuals (i.e. one tenth of the initial population size). We perform 100 runs, where a single run consists of 5000 iterations each projected over 100 years, and calculate the mean fraction of projections that fell below the quasi-extinction threshold during or before the year $t$ (Morris and Doak, 2002).

\section{Results}

285 Addition of the seed bank and demographic uncertainty

286 Inclusion of an unstructured seed bank show fairly small differences in $\lambda$ for most species 287 regardless of the assumptions made (See Fig. 2 and Table 2 to compare the growth rates). 288 However, the potential range of growth rates when demographic parameters are uncertain can be 289 large depending on the species and the amount of temporal variation observed. The 8 tree 290 species, for example, have stable populations $\left(\lambda_{1} \approx 1\right)$ and variation in seed bank parameters has 291 little influence in all of the models with the exception of Illicium anisatum (Schisandraceae).

292 Subsequently, using informed priors for these species has little impact on the p.d.f. of the 293 distribution of possible growth rates. In contrast, the shorter-lived shrub species, Atriplex 294 acanthocarpa (Amaranthaceae) and Sambucus racemosa (Adoxaceae), are more sensitive to the 
introduction of a seed bank and demographic uncertainty in their vital rates. Here, using an informed prior on germination narrows the p.d.f. of the distribution of possible growth rates for the A. acanthocarpa 1996-1997 matrix during an extremely productive year (Fig. 3). Conversely, the use of prior information during the poorer 1997-1999 years appears to increase the spread of possible growth rates. Despite this, poor years tend to have a narrower distribution of possible growth rates and are therefore less sensitive to changes in demographic parameters compared to good years (Figs. 3 and 4). Generally, the p.d.f. of growth rates are skewed, with peaks being concentrated when germination rates are high and greater variation in $\lambda_{1}$ when germination is low (Figs. 3 and 4). However, we note these simulations only investigate the unstructured seed bank scenario. Further uncertainties exist with regards to the longevity and structure of the seed bank, both of which we only briefly explore in this study (Supplementary Table S1).

Elasticity analyses in most plant species, particularly the trees, reveal survival of the adult 307 or late juvenile stages to be the most important demographic process affecting $\lambda_{1}$. The addition of 308 the seed bank did not change this pattern except in the case of A. acanthocarpa during 1997-98 309 and 1998-99 when the populations were declining (Supplementary Table S2). In the 1997-98 310 period, the elasticity of $\lambda_{1}$ to stasis in the third adult stage is drastically reduced $(0.5759$ to 311 0.0647) following the inclusion of the seed bank, whereas the elasticity of $\lambda_{1}$ to stasis in the seed 312 bank is high (0.7919). Similarly, the elasticity of $\lambda_{1}$ to stasis in the first adult stage during the 313 1998-99 period, is reduced from 0.3983 to 0.12 after seed bank addition, while the elasticity of $\lambda_{1}$ 314 to survival in the seed bank is high (0.5039).

\section{Removal of the seed bank and uncertainty in seed bank presence}


Removal of the seed bank further increases population growth when $\lambda_{1}>1$ since germination is no longer delayed, and reduces population persistence when $\lambda_{1}<1$ since the buffering effect is no longer present (Table 3). The notable exception is the wetland herb Helenium virginicum (Asteraceae), where removing the seed bank decreases $\lambda_{1}(1.1145$ to 0.6985$)$ even though the population was increasing. This unusual response is likely a combination of extremely high fecundity into the seed bank and within-the-seed-bank stasis rate (1025 and 0.987 respectively), and the comparatively low fecundity and survival of the smallest stage (0.0075 and 0.199 respectively; see Supplementary File S1 for the original and updated matrices). Decreases in $\lambda_{1}$ is also particularly substantial for Carduus nutans (Asteraceae) and Digitalis purpurea (Plantaginaceae), but is much smaller or unchanged for the remaining species when the seed bank is removed for poor years.

\section{Extinction probabilities}

Inclusion of the seed bank using point estimates for germination generally results in longer times to local extinction (Supplementary Figure S1). Extinction times for A. acanthocarpa for the $330 \quad 1997-98$ and 1998-99 matrices have relatively high increases in extinction times (> 25 years) 331 when the seed bank is included. In addition, certain populations of $A$. canescens and the 332 perennial herb $D$. purpurea are no longer predicted to go extinct in the next 100 years. The 334 times. In one population, however, including the seed bank doubles the time to extinction from 8 335 to 16 years, which is relatively high when compared to the life expectancy of the species $\left(\eta_{E} \cong 2-\right.$ 3363 years, based on the fundamental matrix estimation; Caswell, 2001).

The seed bank generally improves the outlook of stochastic projections for the estimation 338 of extinction probability, but patterns vary across species (Supplementary Figure S2). The 
cumulative probability of extinction at 100 years decreases drastically for A. acanthocarpa from 0.19 to 0.01 following inclusion of the seed bank. The extinction probability for $A$. canescens has an interesting pattern where the matrix model with the seed bank results initially in a higher extinction probability than the original in the first 38 years and eventually in a lower extinction probability, but in the remaining two cases, the seed bank has little effect on the extinction probability. Both populations of D. purpurea are predicted to go extinct within 100 years, however, there is a modest increase in extinction time (16 to 30 years) for one population and a smaller increase in extinction time for the other (4 to 9 years).

\section{Discussion}

The presence of cryptic life stages and their exclusion from demographic models continues to be an impediment to understand and model the population dynamics. These cryptic stages are diverse, ranging from clonality, vegetative dormancy, hibernation, diapause, aestivation, etc., as is their potential impact on population dynamics and model outcomes. Using the seed bank as a well-known example of a cryptic life stage, we demonstrate the use of simulations as a means to reduce uncertainty in seed bank dynamics and to reveal the relative importance of the seed bank and its effect on model outcomes. Ignoring the seed bank in a population can produce variable degrees of uncertainty in the estimates of growth rates and extinction probabilities depending on the observed temporal variation in key vital rates. For example, models with high seedling survival and low temporal variation in demographic parameters are more robust to seed bank uncertainty compared to models with high temporal variation in growth rates and demographic parameters. Furthermore, the potential impact of the seed bank can be influenced by the assumptions underpinning the models and the accuracy of the vital rates that are obtained.

\section{Dealing with the seed bank when its presence is unknown or uncertain}


Anticipating the species for which the seed bank will be an important factor is challenging due to the uncertainty in the demographic parameters of the seed bank stage across species, populations 364 and time. Here, we provide a protocol outlining the different approaches one can take when 365 conducting a plant population study (Fig. 5). First, the potential presence of a seed bank can be determined through a literature search of seed germination and dormancy values (Baskin and Baskin, 2014, is of particular note). Second, if a seed bank in the population is confirmed, the best option would be to obtain field data over the study period to provide estimates for the seed bank vital rates. Without this field data, characterising the potential role of the seed bank becomes exceptionally difficult and assumptions regarding fecundity, seed survival, viability and longevity need to be made. Prior knowledge from the literature can narrow down these possibilities to some extent, although seed characteristics and seed age composition can vary substantially between sites. Also, knowledge from the literature or from field and glasshouse trails may not necessarily reflect those of the study site and must therefore be interpreted with caution (Gross and Mackay, 2014). Finally, simulations can be used to complement these literature estimates to efficiently investigate the numerous possible assumptions regarding seed bank vital rates and determine plausible effects of a potential seed bank. Garcia et al. (2010), for example, performed simulations covering feasible information obtained in the field or from other publications for the long-lived orchid Cypripedium calceolus (Orchidaceae) to justify excluding the seed bank from their models. Likewise, for many of our species, without the benefit of greater observed temporal variation, ignoring the seed bank would appear to be of minimal consequence for the original study, but it is still crucial that an investigation is performed to provide this justification. Otherwise, effective implementation and assessment of management 
strategies will be hindered as the demographic model is potentially missing a key component of the life cycle.

\section{Uncertainty in the seed bank and its effect on model outcomes}

387 Demographic uncertainty in the estimation of vital rates appears to be the major driver in 388 influencing the model predictions but varies by species. Long-lived tree species are virtually 389 unaffected by both the inclusion of the seed bank and variation in their demographic parameters. 390 This is not entirely surprising as high adult survival and reduced mortality when environmental 391 conditions are poor can be an alternative strategy to improve the long-term fitness (Clauss and 392 Venable, 2000). In such species, it is likely that the seed bank will be of little consequence, 393 although it would still be prudent to confirm this suspicion (e.g., using simulations) before 394 ignoring the seed stage. Additionally, this does not necessarily mean the seed bank will be 395 unimportant if the population were to experience a large disturbance. In contrast, in populations 396 experiencing greater fluctuations in demographic parameters and population growth rates, the 397 contribution of the seed bank is more visible as is evident for A. acanthocarpa, where previously 398 high elasticities for juvenile and adult survival shift towards survival in the seed bank during 399 poor demographic years (Table S2).

400 Temporal variation and the type of demographic year are also factors that will affect of 401 demographic uncertainty. Alterations in germination and viability rates during poor demographic 402 years $\left(\lambda_{1}<1\right)$ have relatively little impact on the growth rate compared to favourable years when $403 \lambda_{1}$ exceeded 1 (Fig. 4). Large reproductive failures during poor years would have resulted in low 404 seed bank and seedling input, reducing the impact of varying seed bank vital rates on $\lambda_{1}$. In 405 contrast, we would expect variations in germination and viability rates during poor years to have a much larger impact on long-term persistence and environmental stochasticity. Nonetheless, 
with the inclusion of prior information from the literature, the p.d.f. of the distribution of growth rates during these poor demographic years shifts towards one (Fig. 3). We note however that this is assuming dormancy is higher (low germination) during poor demographic years and lower during productive years (Kalisz and McPeek, 1992), but seed dormancy needs not necessarily be correlated with fitness. Interestingly, the use of literature did not necessarily reduce uncertainty 412 in the range of possible growth rates and in fact becomes more variable when dormancy is high 413 (Fig. 3). This is an important result as an uninformed simulation may underestimate the level of 414 uncertainty in demographic output if prior information suggests potentially high levels of 415 dormancy. In contrast, when dormancy is suspected to be low, the impact of the seed bank will 416 not be as great, thus variation in its demographic parameters will be of much less consequence.

417 In addition to variations in demographic parameters, changes to how the seed bank is 418 included can impact the model structure and outcomes. Our uncertainty simulations investigate a 419 1-year seed bank as a counterfactual example to illustrate the need for including a seed bank. We acknowledge that while single point estimates of $\lambda_{1}$ under the unstructured scenario showed 421 minor increases compared to the original models, this gradually reduces when additional age 422 classes are introduced (See Table S1 to compare the growth rates). This is likely because a 423 higher proportion of emergent seedlings are now assumed to have germinated from seeds 424 produced in previous years, thus compounding the effect of delayed germination. Similarly, if we 425 assume seeds do not survive beyond the first, second or third year, the effect of the seed bank is 426 almost negligible (See Table S1). However, such estimates are overly conservative and most 427 models that do include the seed bank do not make this assumption.

428 Another interesting scenario that can be tested is that of no seed bank. Our removal simulations provides insights into the worst-case scenarios when the seed bank is ignored despite 
being crucial to persistence (as indicated by high elasticities to seed bank transitions). As expected, the implications of ignoring the seed bank in these cases were generally much more evident with decreases in $\lambda_{1}$ of up to 0.4 , although these reflect the worst-case scenarios and changes in $\lambda_{1}$ would not generally be so dramatic. The assumption of fecundity being equal to that of the previous year $\left(F_{j}(t)=F_{j}(t)\right)$ was necessary due to lack of information, however, interannual variation is likely to play an important role for many species. When variance in the fecundity is high, the contribution of the seed bank would be overestimated in good demographic years and underestimated in bad demographic years. This would likely have similar consequences on any changes in $\lambda_{1}$, thus we might expect the benefit of having a seed bank during poor years to also be underestimated.

Analysis of extinction probabilities over shorter time periods can provide additional insights not considered under asymptotic analyses. Comparing the difference in extinction times and cumulative extinction probabilities between models with and without the seed bank can provide an empirical measure of the uncertainty in population persistence when the seed bank is 444 ignored, and this measure can vary substantially between years and species. Although increases 445 in extinction times can appear trivial, they must also be considered in context of the life 446 expectancy. For example, the seed bank had little effect on the times to extinction for the 447 populations of $C$. nutans. However, given the short life expectancy of this species, even a small 448 increase in extinction time may prove crucial.

\section{Conclusions}

450 There is still much progress to be made concerning the seed bank and it is but one of many 451 cryptic life stages with the potential to create biases in our model outputs. Adult plants, for 452 example, can undergo vegetative dormancy, whereby individuals do not sprout above ground for 
one or more growing seasons, resulting in overestimated mortality rates and incorrect assignment of false birth events to re-emerging individuals (Logofet et al., 2019, Shefferson, 2009).

Demographic studies on animals can be additionally problematic as certain stages of the life cycle may occupy inaccessible locations or be absent from sampling designs. Katzner et al. (2011) found raptor populations to be drastically underestimated as monitoring programs only captured the breeding portion of the population. In both plant and animal studies, incorrect estimates of population size, persistence, and occupancy in demographic models can result in suboptimal management decisions and make it difficult to assess the success of these management actions (Regan et al., 2011). While we have focused exclusively on plant population models in this study, characterising the extent of exclusion of cryptic life stages in demographic models for animals is just as important and open for investigation with the availability of the COMADRE Animal Matrix Database (Salguero-Gómez et al., 2016).

\section{Acknowledgements}

The authors would like to acknowledge the critical review by an anonymous reviewer that has 467 greatly improved the manuscript, E. Crone and J. Ehrlén for helpful comments in previous 468 versions of this manuscript, and S. Ramula and members of the ARC-NZ Research Network for 469 Vegetation Function Plant Population Syndromes working group.

\section{Author Contributions}

472 The first and last author conceived and designed the study. The first author collected the data and 473 performed the analysis. All authors contributed to the study methodology and writing of the 474 manuscript. 


\section{References}

Abe S, Motai H, Tanaka H, Shibata M, Kominami Y, Nakashizuka T (2008). Population maintenance of the short-lived shrub Sambucus in a deciduous forest. Ecology, 89, 11551167.

Adams VM, Marsh DM, Knox JS (2005). Importance of the seed bank for population viability and population monitoring in a threatened wetland herb. Biol. Conserv., 124, 425-436.

Angert AL (2006). Demography of central and marginal populations of monkey flowers (Mimulus cardinalis and M. lewisii). Ecology, 87, 2014-2025.

Baskin CC, Baskin GM (2014). Seeds: Ecology, biogeography, and evolution of dormancy and germination, Academic Press, San Diego, California.

Burns JH, Blomberg SP, Crone EE, Ehrlén J, Knight TM, Pichancourt JP, Ramula S, Wardle GM, Buckley YM (2010). Empirical tests of life-history evolution theory using phylogenetic analysis of plant demography. J. Ecol., 98, 334-344.

Caswell H (2001). Matrix population models: construction, analysis and interpretation, Sinauer Associates, Inc., Sunderland, Massachusetts.

Chien PD, Zuidema PA, Nghia NH (2008). Conservation prospects for threatened Vietnamese tree species: results from a demographic study. Popul. Ecol., 50, 227-237.

Clauss MJ, Venable DL (2000). Seed germination in desert annuals: an empirical test of adaptive bet hedging. Am. Nat., 155, 168-186.

COMPADRE Plant Matrix Database. 2019. COMPADRE Plant Matrix Database v5.0.0. [Online]. Max Planck Institute for Demographic Research (Germany). [Accessed 20th May 2019].

Crone EE, Menges ES, Ellis MM, Bell T, Bierzychudek P, Ehrlén J, Kaye TN, Knight TM, Lesica P, Morris WF, Oostermeijer G, Quintana-Ascencio PF, Stanley A, Ticktin T, Valverde T, Williams JL (2011). How do plant ecologists use matrix population models? Ecol. Lett., 14, 1-8.

de Kroon H, Plaisier A, J.V. G (1987). Density dependent simulation of the population dynamics of a perennial grassland species, Hypochaeris radicata. Oikos, 50, 3-12.

de Kroon H, van Groenendael J, Ehrlen J (2000). Elasticities: a review of methods and model limitations. Ecology (Wash. D. C.), 81, 607-618.

Doak DF, Thomson D, Jules ES (2002). Population viability analysis for plants: understanding the demographic consequences of seed banks for population health. In: Beissinger SR, McCullough DR (eds.) Population Viability Analysis. The University of Chicago Press, Chicago, Illinois, USA.

Fenner M (2000). Seeds: the ecology of regeneration in plant communities, CABI Publishing, Wallingford, UK.

Garcia MB, Goni D, Guzman D (2010). Living at the edge: local versus positional factors in the long-term population dynamics of an endangered orchid. Conserv. Biol., 24, 1219-1229.

Geiser F (2004). Metabolic rate and body temperature reduction during hibernation and daily torpor. Annu. Rev. Physiol., 66, 239-274.

Gremer JR, Crone EE, Lesica P (2012). Are dormant plants hedging their bets? Demographic consequences of prolonged dormancy in variable environments. Am. Nat., 179, 315-327.

Gross C, Mackay D (2014). Two decades of demography reveals that seed and seedling transitions limit population persistence in a translocated shrub. Annals of Botany.

Harper JL (1977). Population biology of plants, Academic Press, London. 
Jongejans E, Shea K, Sheppard AW (2006). What controls the population dynamics of the invasive thistle Carduus nutans in its native range? J. Appl. Ecol., 43, 877-886.

Kalisz S, McPeek MA (1992). Demography of an age-structured annual: resampled projection matrices, elasticity analyses, and seed bank effects. Ecology, 73, 1082-1093.

Katzner TE, Ivy JAR, Bragin EA, Milner-Gulland E, DeWoody JA (2011). Conservation implications of inaccurate estimation of cryptic population size. Anim. Conserv., 14, 328332.

Kaye TN, Pyke DA (2003). The effect of stochastic technique on estimates of population viability from transition matrix models. Ecology, 84, 1464-1476.

Kisanuki H, Nakasu M, Nakai A, Yurugi Y (2008). Predicting the population dynamics of three understory broad-leaved evergreen species under the influence of Sika deer in primary and secondary forests of mid-western Japan. J. For. Res., 13, 52-58.

Kouassi KI, Barot S, Gignoux J, Bi IAZ (2008). Demography and life history of two rattan species, Eremospatha macrocarpa and Laccosperma secundiflorum, in Cote d'Ivoire. J. Trop. Ecol., 24, 493-503.

Logofet DO, Kazantseva ES, Belova IN, Onipchenko VG (2019). Disappointing viability forecat for a local population of Androsace albana in a random environment. Zh. Obshch. Biol., $80,1-13$.

Mondragon D (2009). Population viability analysis for Guarianthe aurantiaca, an ornamental epiphytic orchid harvested in Southeast Mexico. Plant Species Biol., 24, 35-41.

Morris WF, Doak DF (2002). Quantitative conservation biology: theory and practice of population viability analysis, Sinauer Associates, Sunderland, Massachusetts.

Parker IM (2000). Invasion dynamics of Cytisus scoparius: a matrix model approach. Ecol. Appl., 10, 726-743.

Quitete Portela RDC, Bruna EM, Maes dos Santos FA (2010). Demography of palm species in Brazil's Atlantic forest: a comparison of harvested and unharvested species using matrix models. Biodivers. Conserv., 19, 2389-2403.

R Core Team. 2015. R: A language and environment for statistical computing [Online]. Vienna, Austria: R Foundation for Statistical Computing. [Accessed 3rd October 2018].

Regan TJ, Chades I, Possingham HP (2011). Optimally managing under imperfect detection: a method for plant invasions. J. Appl. Ecol., 48, 76-85.

Salguero-Gómez R, Jones OR, Archer CR, Buckley YM, Che-Castaldo J, Caswell H, Hodgson D, Scheuerlein A, Conde DA, Brinks E, de Buhr H, Farack C, Gottschalk F, Hartmann A, Henning A, Hoppe G, Römer G, Runge J, Ruoff T, Wille J, Zeh S, Davison R, Vieregg D, Baudisch A, Altwegg R, Colchero F, Dong M, de Kroon H, Lebreton J-D, Metcalf CJE, Neel MM, Parker IM, Takada T, Valverde T, Vélez-Espino LA, Wardle GM, Franco M, Vaupel JW (2015). The COMPADRE Plant Matrix Database: an open online repository for plant demography. J. Ecol., 103, 202-218.

Salguero-Gómez R, Plotkin JB (2010). Matrix dimensions bias demographic inferences: implications for comparative plant demography. Am. Nat., 176, 710-722.

Shefferson RP (2009). The evolutionary ecology of vegetative dormancy in mature herbaceous perennial plants. J. Ecol., 97, 1000-1009.

Sletvold N, Rydgren K (2007). Population dynamics in Digitalis purpurea: the interaction of disturbance and seed bank dynamics. J. Ecol., 95, 1346-1359.

Stöcklin J, Fischer M (1999). Plants with longer-lived seeds have lower local extinction rates in grassland remnants 1950-1985. Oecologia, 120, 539-543. 
Tanner JE (2001). The influence of clonality on demography: patterns in expected longevity and survivorship. Ecology, 82, 1971-1981.

Venable DL (2007). Bet hedging in a guild of desert annuals. Ecology, 88, 1086-1090.

Verhulst J, Montana C, Mandujano MC, Franco M (2008). Demographic mechanisms in the coexistence of two closely related perennials in a fluctuating environment. Oecologia (Berl.), 156, 95-105.

Vitalis R, Glémin S, Olivieri I (2004). When genes go to sleep: the population genetic consequences of seed dormancy and monocarpic perenniality. The American Naturalist, 163, 295-311.

Warner RR, Chesson PL (1985). Coexistence mediated by recruitment fluctuations: a field guide to the storage effect. Am. Nat., 125, 769-787. 
4579 Tables

580 Table 1. Comparison of studies from the MPM2008-2011 database with a previous review by

(Doak et al., 2002) examining the inclusion of the seed bank in plant population models. Studies

582 were classified as justified exclusion if a valid reason was provided for not including the seed

583 bank (e.g. the authors found no evidence of a seed bank) and unjustified exclusion if the

584 possibility of a seed bank was not explored or mentioned. Studies from COMPADRE were not

585 analysed to determine whether the exclusion of the seed bank was justified.

\begin{tabular}{lccc}
$\begin{array}{l}\text { Population models and seed } \\
\text { banks }\end{array}$ & $\begin{array}{c}\text { Doak et al. } \\
\mathbf{( 2 0 0 2 )}\end{array}$ & $\begin{array}{c}\text { MPM2008- } \\
\mathbf{2 0 1 1}\end{array}$ & $\begin{array}{c}\text { COMPADRE } \\
\text { v.5.0.0 }\end{array}$ \\
\hline Included & $34(48.6 \%)$ & $16(29.1 \%)$ & $292(34.0 \%)$ \\
Justified exclusion & $6(8.6 \%)$ & $13(23.6 \%)$ & \\
Unjustified exclusion & $30(42.9 \%)$ & $26(47.3 \%)$ & $568(66.0 \%)$ \\
\hline
\end{tabular}


Table 2. Comparison of population growth rates $\left(\lambda_{1}\right)$ following the addition of a seed bank. Pop. refers to populations of the same species in the same study. Avg. represents the growth rate from the mean matrix for each population. Stoch. represents the mean stochastic growth rate from populations projected over 10,000 times. SB denotes single point estimates from models with germination and viability equal to that obtained from the literature. Growth rates from Monte Carlo simulations were obtained from the mean of 10,000 trials with parameters for germination, viability, seed survival, and seedling survival drawn from uniform distributions when priors are uninformed, while germination and viability were drawn from beta distributions when the priors were informed. $95 \%$ confidence intervals for Monte Carlo simulations and stochastic growth rates are displayed in round brackets.

\section{Life}

Study

Single time period

Chien et al. (2008) Calocedrus macrolepus Parashorea chinensis Pinus kwangtungensis

Tree
Tree
Tree

Kisanuki et al. (2008) Illicium anisatum

Abe et al. (2008)

Sambucus racemosa

$\begin{array}{ccccc}\text { Tree } & 1 & 1 & 1.1271 & 1.1647 \\ & 2 & 1 & 1.1864 & 1.2276 \\ & & & & \\ \text { Shrub } & 1 & 1 & 1.1696 & 1.1690 \\ & 2 & 1 & 1.0007 & 1.0012 \\ & 3 & 1 & 2.2227 & 2.2031\end{array}$

0.9792
0.9955

0.9786

0.9948
0.9776

1.0012

2031

0.9811
Monte Carlo sim. uninformed prior

$0.9700(0.9683,0.9785)$ $0.9963(0.9948,1.0040)$ $0.9786(0.9776,0.9834)$

Monte Carlo sim. informed prior

$0.9699(0.9683,0.9752)$ $0.9954(0.9948,0.9978)$

$0.9782(0.9776,0.9799)$

$1.1344(1.1258,1.1765) \quad 1.1408(1.1263,1.1778)$ $1.1931(1.1828,1.2363) \quad 1.2008(1.1840,1.2416)$

$1.1789(1.1661,1.2355) \quad 1.1951(1.1674,1.2639)$

$1.0109(1.0007,1.0622) \quad 1.0255(1.0008,1.0963)$

$2.1588(2.0468,2.2210) \quad 2.1459(2.0551,2.2125)$

$0.9806(0.9792,0.9887)$

$0.9805(0.9792,0.9842)$ 
Quitete Portela et al. (2010)

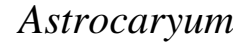

Atriplex canescens

$\begin{array}{llll}\text { Tree } & 1 & 1 & 0.9606\end{array}$

0.9661

$0.9638(0.9607,0.9815)$

$0.9621(0.9607,0.9658)$

\section{Multiple time periods}

Mondragon (2009)

Guarianthe aurantiaca

$\begin{array}{ccccc}\text { Herb } & 1 & 1 & 0.9851 & 0.9881 \\ & 1 & 2 & 0.9895 & 0.9908 \\ & & \text { Avg. } & 0.9869 & 0.9891 \\ & & \text { Stoch. } & 0.9869 & 0.9891 \\ & & & (0.9868,0.9869) & (0.9891,0.9892)\end{array}$

Geonoma schottiana

Tree

$\begin{array}{lll}1 & 1 & 1.0093 \\ 1 & 1 & 2\end{array}$

Avg.

Stoch.

Tree

1
2
Avg.
Stoch.$$
(0.9998,0.9998)
$$

1.0043
0.9823
0.9949

$$
\begin{aligned}
& 1.0106 \\
& 0.9968 \\
& 1.0085 \\
& 1.0005
\end{aligned}
$$

$(1.0005,1.0005)$

$$
1.0240
$$

1.0002

1.0150

Stoch.

1.0050

1.0146

$(1.0050,1.0050) \quad(1.0145,1.0148)$

Atriplex acanthocarpa shos

1
1

1
2
3
Avg.
Stoch.

$\begin{array}{cc}2.4526 & 2.3455 \\ 0.7301 & 0.8305 \\ 0.7436 & 0.8862 \\ 1.3461 & 1.3351 \\ 1.1486 & 1.1792 \\ 1460,1.1512) & (1.1773,1.1811) \\ 1.7211 & 1.7012 \\ 0.9451 & 0.9451 \\ 0.8805 & 0.8835 \\ 1.1346 & 1.1648 \\ 1.0775 & 1.1140 \\ 0730,1.0779) & (1.1120,1.1159)\end{array}$

$\begin{array}{ll}0.9917(0.9851,1.0253) & 0.9872(0.9851,0.9929) \\ 0.9929(0.9895,1.0107) & 0.9905(0.9895,0.9931)\end{array}$

$1.0111(1.0093,1.0209) \quad 1.0100(1.0093,1.0112)$

$0.9971(0.9964,1.0001) \quad 0.9966(0.9964,0.9972)$

$-$

$1.0114(1.0050,1.0442)$

$1.0115(1.0050,1.0453)$

$1.0213(1.0050,1.0712)$

$1.0216(1.0050,1.0721)$

$2.3016(2.0678,2.4476)$ $0.7418(0.7302,0.8288)$

$0.7712(0.7438,0.8951)$

$2.3541(2.0759,2.4489)$ $0.7508(0.7303,0.8362)$ 0.7924 (0.7444, 0.9051)

$1.6883(1.6184,1.7315)$

$0.9451(0.9451,0.9453)$

$0.8810(0.8805,0.8823)$

$1.6791(1.6160,1.7175)$

$0.9451(0.9451,0.9451)$

$0.8805(0.8805,0.8807)$ $\begin{array}{ccc}\text { Avg. } & 1.1346 & 1.1648 \\ \text { Stoch. } & 1.0775 & 1.1140 \\ & (1.0730,1.0779) & (1.1120,1.1159)\end{array}$ 594 
shown in brackets.

\section{Study}

Life Form

\section{Pop. Period}

SB

No variability reported

Adams et al. (2005) Helenium virginicum

de Kroon et al. (1987) Hypochaeris radicata

Herb

$1 \quad 1$

1.1145

0.6985

Variability, single time period

Jongejans et al. (2006)

Carduus nutans

Herb

Herb

11

0.2973

0.2154

Multiple time periods

\section{Angert (2006)}

Mimulus lewisii

Herb

1
2
3

0.4680
0.6270
0.6020




\section{Parker (2000)}

Cytisus scoparius

$\begin{array}{cc}2 & 3 \\ 2 & \text { Avg. } \\ 2 & \text { Stoch. }\end{array}$

$\begin{array}{cc}3 & 1 \\ 3 & 2 \\ 3 & 3 \\ 3 & \text { Avg. } \\ 3 & \text { Stoch. }\end{array}$

$\begin{array}{ll}4 & 1 \\ 4 & 2 \\ 4 & 3\end{array}$

4

Herb

\begin{tabular}{cc}
1.3203 & 1.3746 \\
1.0158 & 1.0410 \\
0.9642 & 0.9949 \\
$(0.9769,0.9787$ & $(0.9935,0.9963)$ \\
1.1052 & 1.1094 \\
1.1197 & 1.1450 \\
0.9523 & 0.9507 \\
1.0510 & 1.0583 \\
1.0472 & 1.0537 \\
$(1.0470,1.0500)$ & $(1.0544,1.0557)$ \\
1.5841 & 1.6704 \\
1.0459 & 1.0470 \\
1.1227 & 1.3083 \\
1.3112 & 1.3448 \\
1.2919 & 1.3198 \\
$(1.2908,1.2930)$ & $(1.3192,1.3205)$ \\
& \\
2.1963 & 2.3331 \\
2.2629 & 2.3858 \\
2.5019 & 2.7128 \\
2.4919 & 2.6927 \\
$(2.4879,2.4960)$ & $(2.6868,2.6986)$ \\
1.7097 & 1.7407 \\
1.2542 & 1.2406 \\
1.5681 & 1.5552 \\
1.5507 & 1.5351 \\
$(1.5495,1.5518)$ & $(1.5343,1.5359)$ \\
1.0040 & 1.0040 \\
0.9295 & 0.9287 \\
0.9748 & 0.9746 \\
0.9740 & 0.9738 \\
$(0.9734,0.9746)$ & $(0.9700,0.9776)$ \\
& \\
\hline &
\end{tabular}


Sletvold and Rydgren (2007)

Digitalis purpurea

Herb

$\begin{array}{cc}1 & 1 \\ 1 & 2 \\ 1 & \text { Avg. } \\ 1 & \text { Stoch. }\end{array}$

$\begin{array}{ll}2 & 1 \\ 2 & 2\end{array}$

2 Avg.

2 Stoch.

$\begin{array}{cc}0.7533 & 0.3174 \\ 0.7831 & 0.5536 \\ 0.7715 & 0.5071 \\ 0.7713 & 0.5008 \\ (0.7712,0.7713) & (0.5002,0.5013) \\ 0.6831 & 0.2983 \\ 1.0198 & 0.9379 \\ 0.8565 & 0.6701 \\ 0.8388 & 0.5794 \\ (0.8384,0.8392) & (0.5787,0.5802)\end{array}$


601 Fig. 1. Life cycle graphs corresponding to: A) a plant life cycle consisting of two stages: the 602 seedlings, Se, and the adults, Ad; B) a plant life cycle consisting of seeds that have remained in 603 the seed bank for 1 year, S1, up to $n$ years, Sn (Eq. 1), and C) a plant life cycle with an 604 unstructured seed bank, SB, (Eq. 2). The transition rate $f_{\mathrm{Se}, \text { Ad }}$ gives the fecundity into the seedling 605 stage while $f_{\mathrm{S} 1, \mathrm{Ad}}$ and $f_{\mathrm{SB}, \mathrm{Ad}}$ gives the fecundity into the seed bank. D) Details of the derivation of 606 Eq. 1 in which the parameters are contained in boxes (see formal notation in the text). Tracking 607 the vital rates required to reach the seedling stage from the adult stage either directly or via the 608 seed bank demonstrates how each term in Eq. 1 was derived.

Fig. 2. Scatterplot comparing population growth rates between models when the seed bank is included (y-axis) and when the seed bank is not included (x-axis). "Addition" refers to models in which we added the seed bank the model (Table 2), while "Removal" refers to models where we removed the seed bank (Table 3). Growth rates for models where the seed bank was added were taken from Monte Carlo simulations with germination prior guided by the literature. Points above the one-to-one line indicate the seed bank has a positive effect on $\lambda$ while points below indicate a negative effect. Points within the red section are declining populations $\left(\lambda_{1}<1\right)$ while those outside are increasing populations $\left(\lambda_{1}>1\right)$.

Fig. 3. Probability density function of the distribution of population growth rates for Atriplex acanthocarpa from A) 1996-1997, B) 1997-1998, and C) 1998-1999 based on 10,000 Monte Carlo simulations calculated using an uninformed prior on germination (uniform distribution) and an informed prior on germination (beta distribution with the mode equal to the germination 
rate obtained from the literature). Red dashed lines indicate the mean growth rate using uninformed and informed priors as reported in Table 2.

Fig. 4. Population growth rate $\left(\lambda_{1}\right)$ as a function of germination rate from the seed bank for $\mathbf{A}$ ) Atriplex acanthocarpa, B) Atriplex canescens, C) Illicium anisatum, and D) Geonoma 628 schottiana. Population growth rates were calculated at seed viability rates of 1 (green line), 0.5 629 (blue line), and 0.1 (red line) for the germination rates between 0 and 1 at intervals of 0.01 . The 630 germination rate for G. schottiana is restricted between 0 and 0.69 to not exceed the total seed 631 survival estimate obtained from the literature.

633 Fig. 5. Flow chart describing different approaches to dealing with a potential seed bank when 634 modelling plant population dynamics. When the presence of the seed bank is uncertain, it is 635 advised that researchers explore this possibility either through literature confirmation, or 636 investigating the soil reserves for viable seeds. By not investigating, the exclusion is unjustified, 637 resulting in the highest possible uncertainty. Justified exclusion, by either proving it does not 638 exist or showing it is unimportant via simulations, gives the highest level of certainty in the 639 model outcomes. Including the seed bank, either by using the literature or data obtained in the 640 field would unavoidably result in some amount of uncertainty from estimating the vital rates, but 641 is preferable to unjustified exclusion. 
642 Fig. 1

A

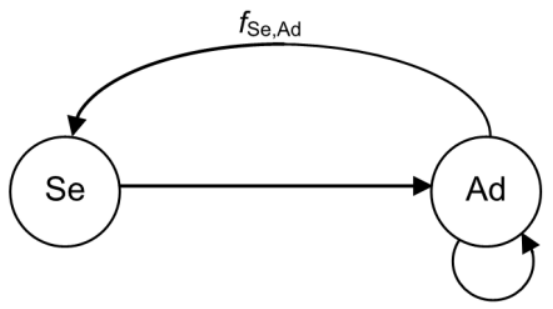

12

13

14

15

B

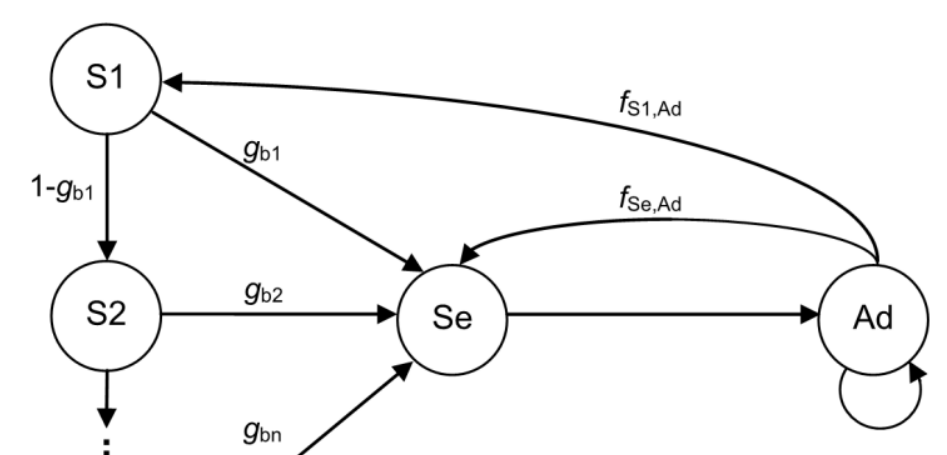

C

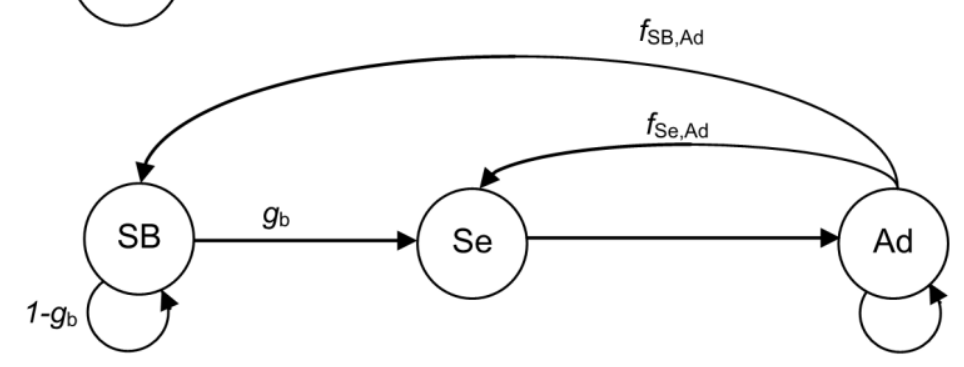

643

D

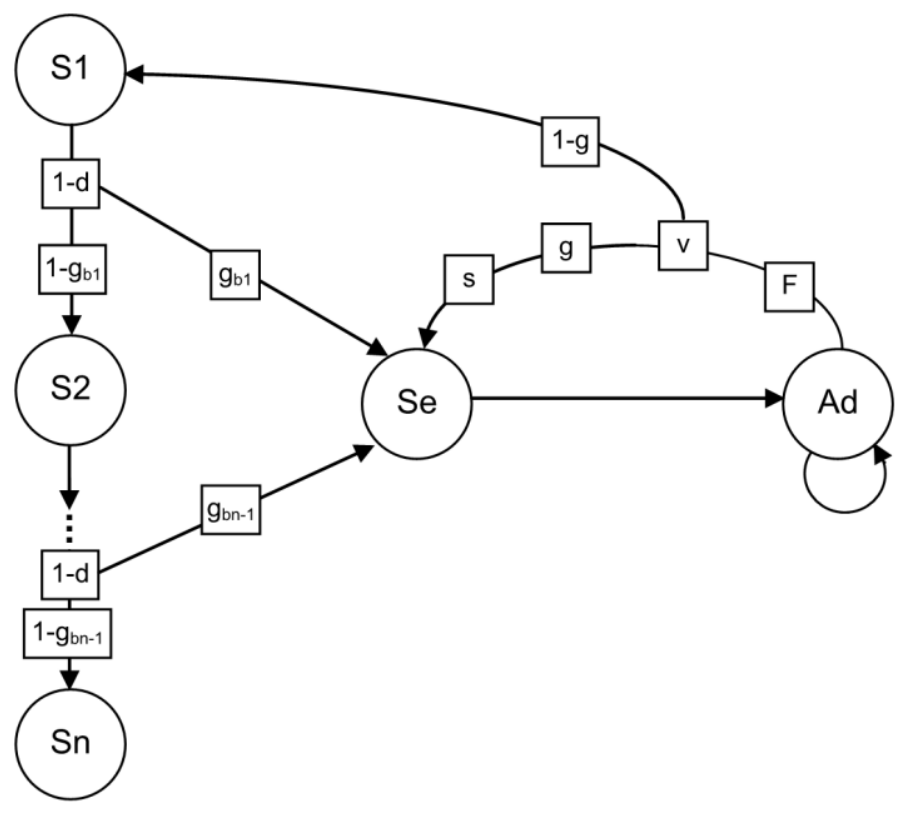




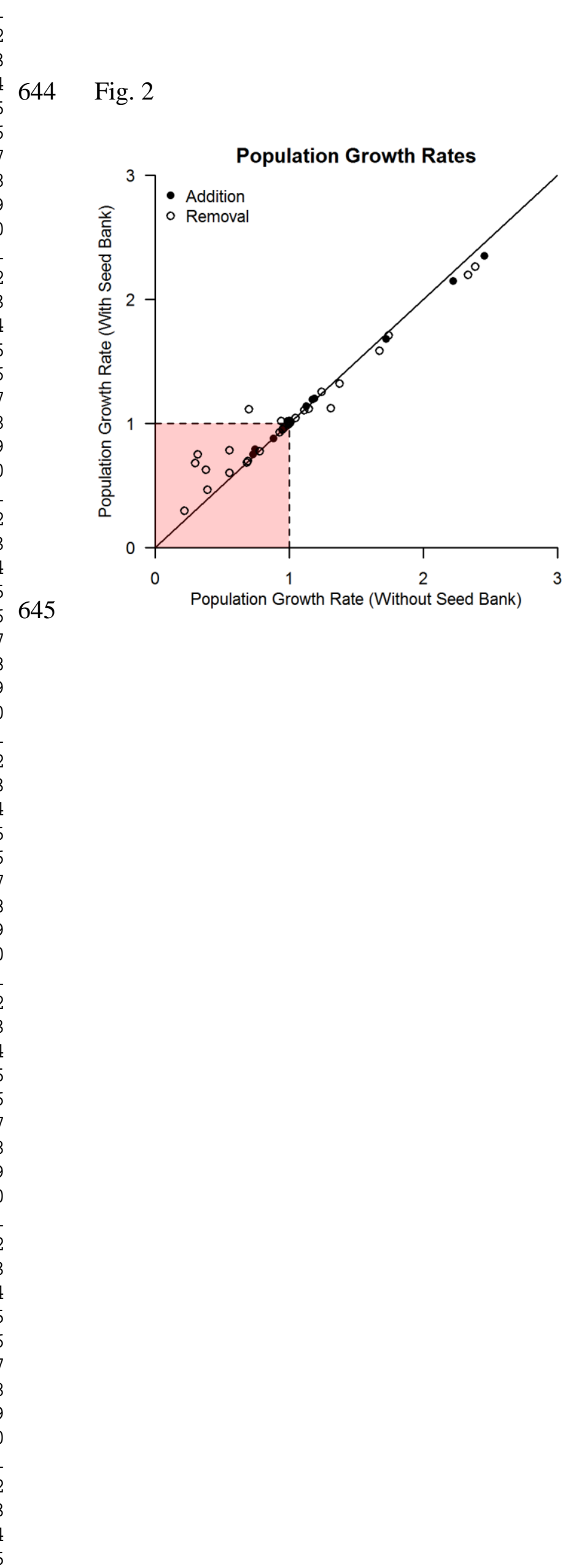



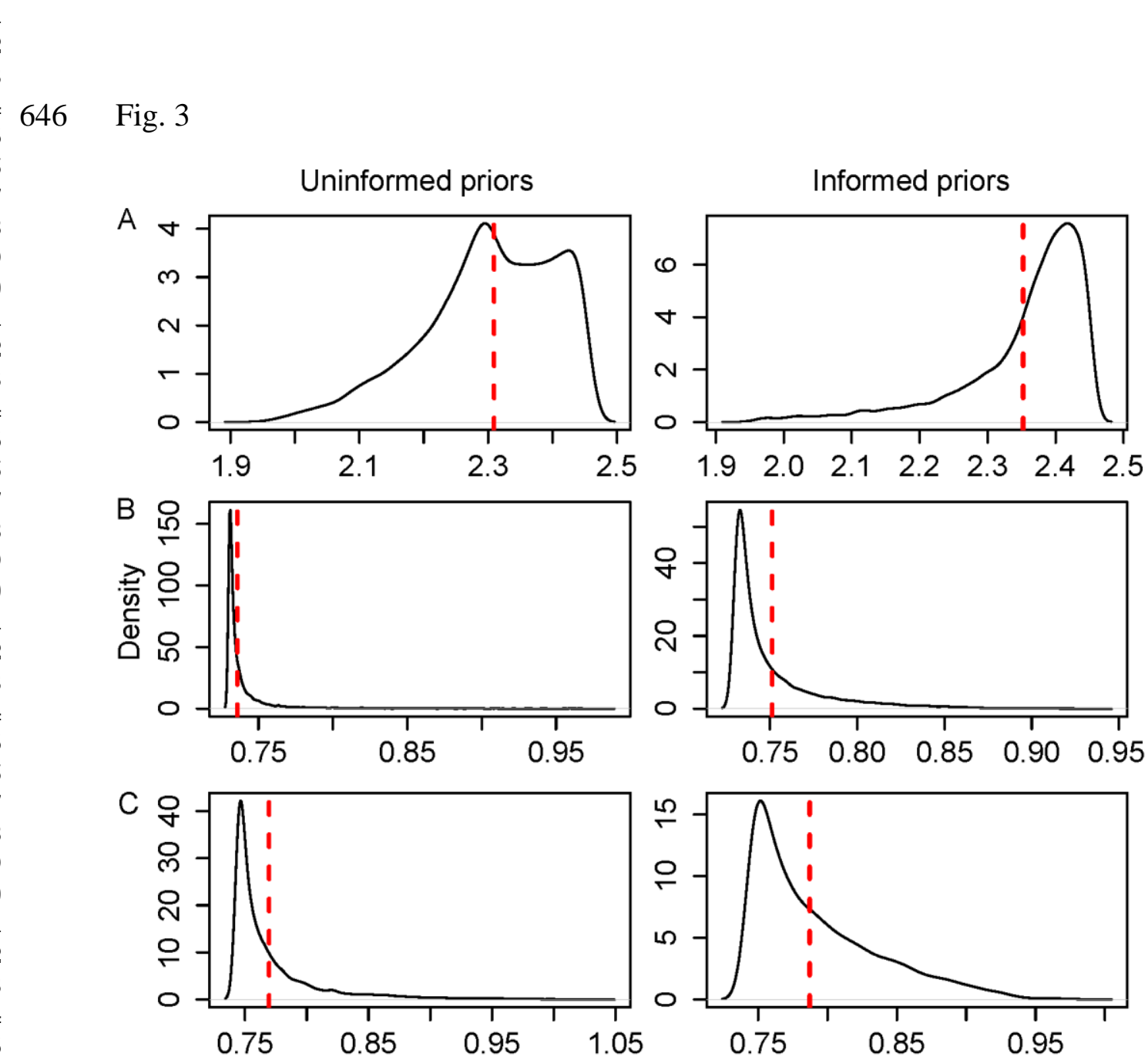
$\begin{array}{lll}4 & 648 & \text { Fig. } 4\end{array}$

6

7

8

9

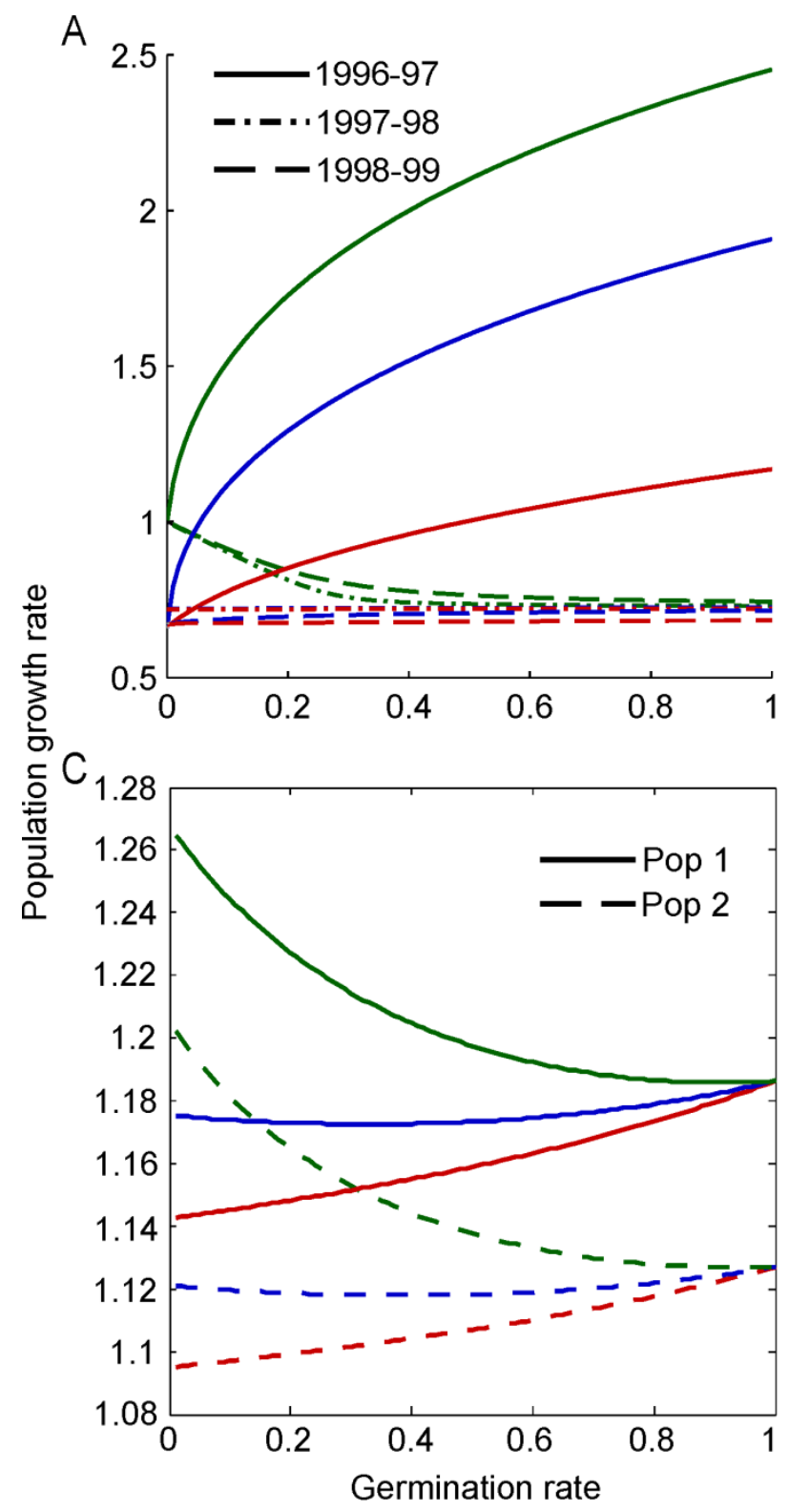

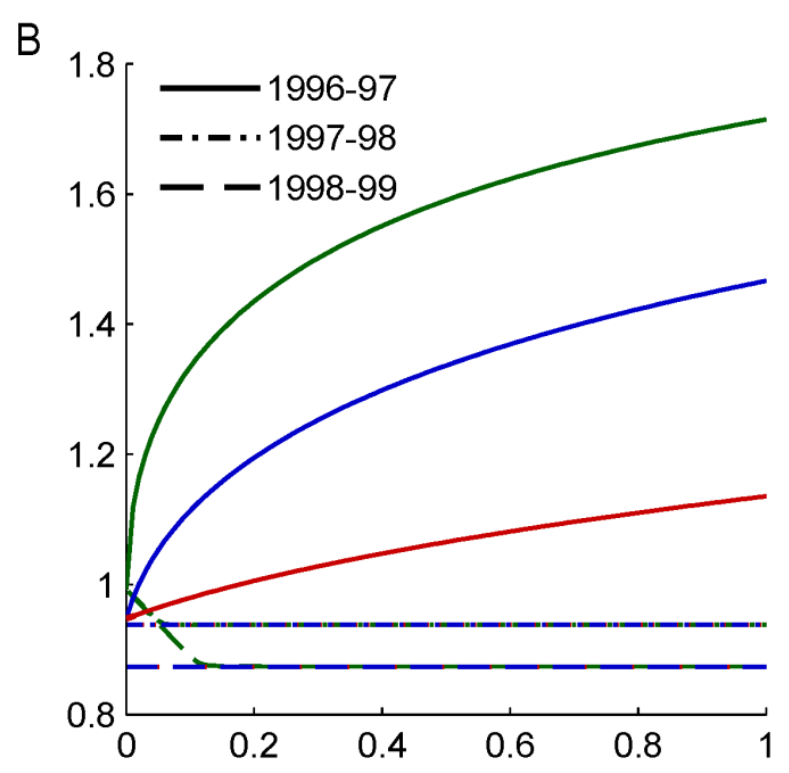

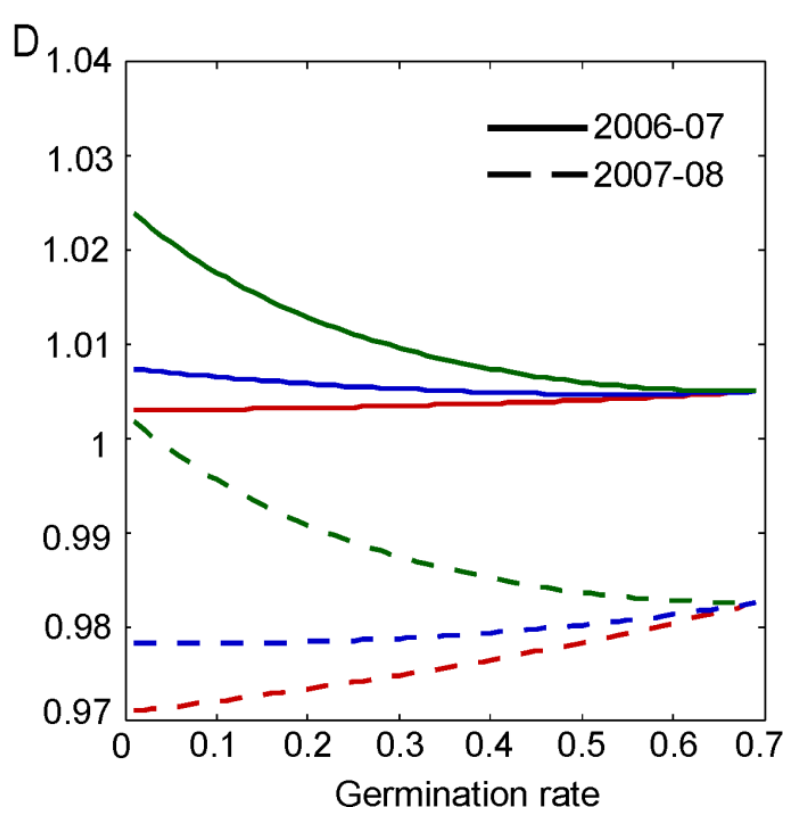


Fig. 5

\section{Source of uncertainty}

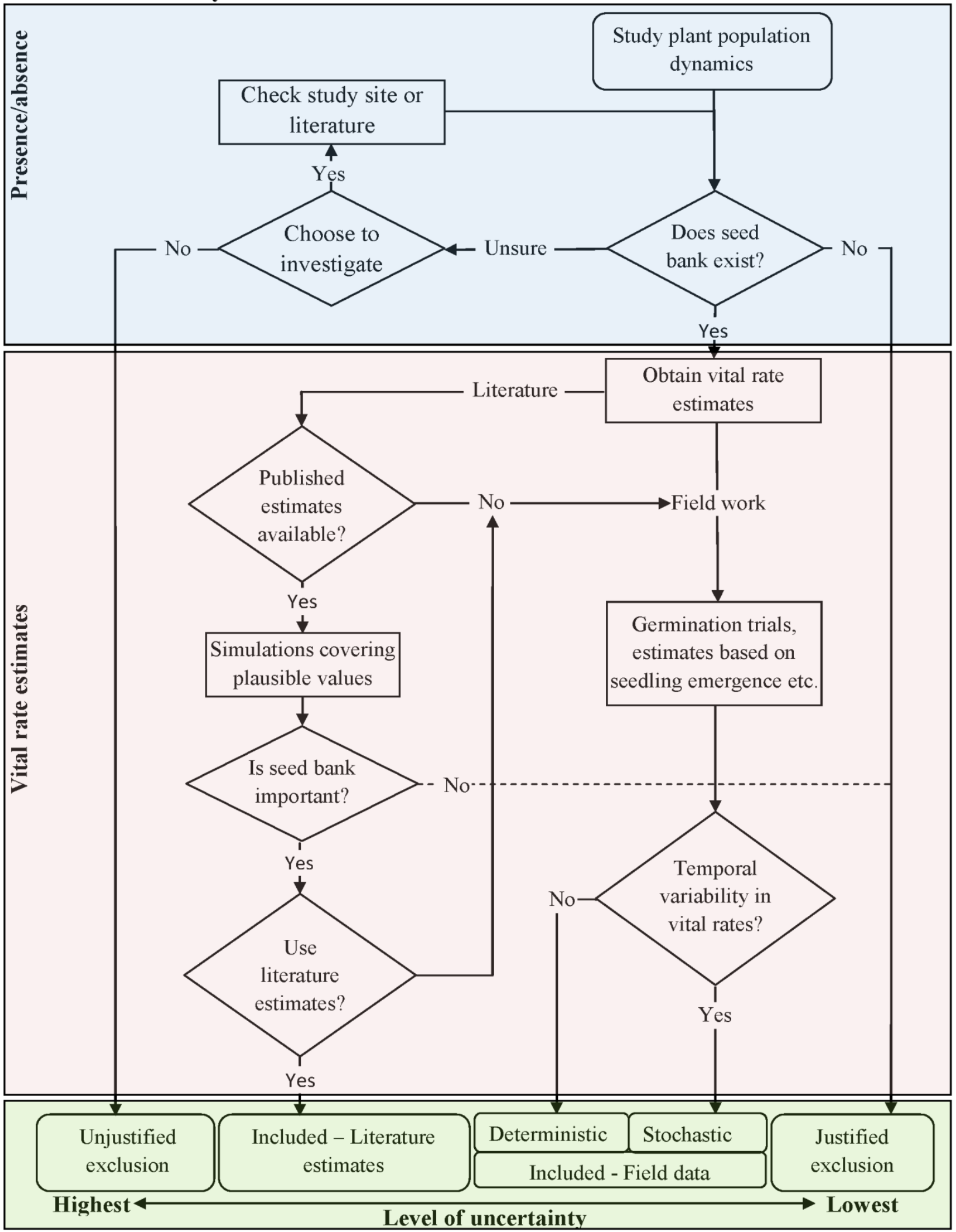


4653 Supporting Information

6

7654 Table S1

8

9655 Population growth rates from seed bank models, Monte Carlo simulations and alternative

656 formulations with 2 and 3 year longevity

\section{Table $\mathbf{S 2}$}

658 Elasticity matrices for 1997 and 1998 for Atriplex acanthocarpa with and without the seed bank

\section{$659 \quad$ Figure $\mathbf{S 1}$}

660 Log population size projections for single point matrices with and without the seed bank

$661 \quad$ Figure $\mathbf{S 2}$

662 Cumulative extinction probabilities of quasi-extinction

\section{Method S1}

664 Example demonstrating methodology for including the seed bank stage and alternative

665 formulations

666 Method S2

667 Example of seed bank stage removal from a matrix model for Digitalis purpurea

\section{$668 \quad$ File $\mathbf{S 1}$}

669 Excel file containing original and updated matrices used for addition and removal simulations 
Click here to download high resolution image

A

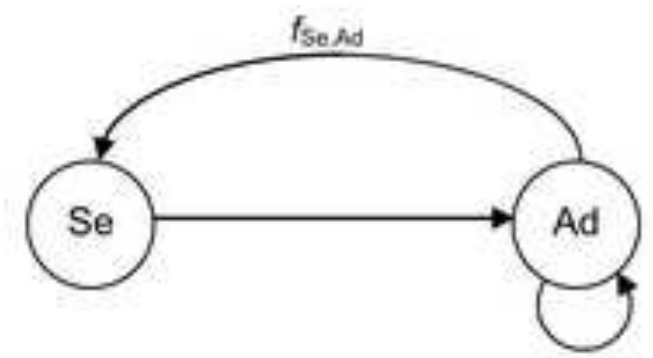

B

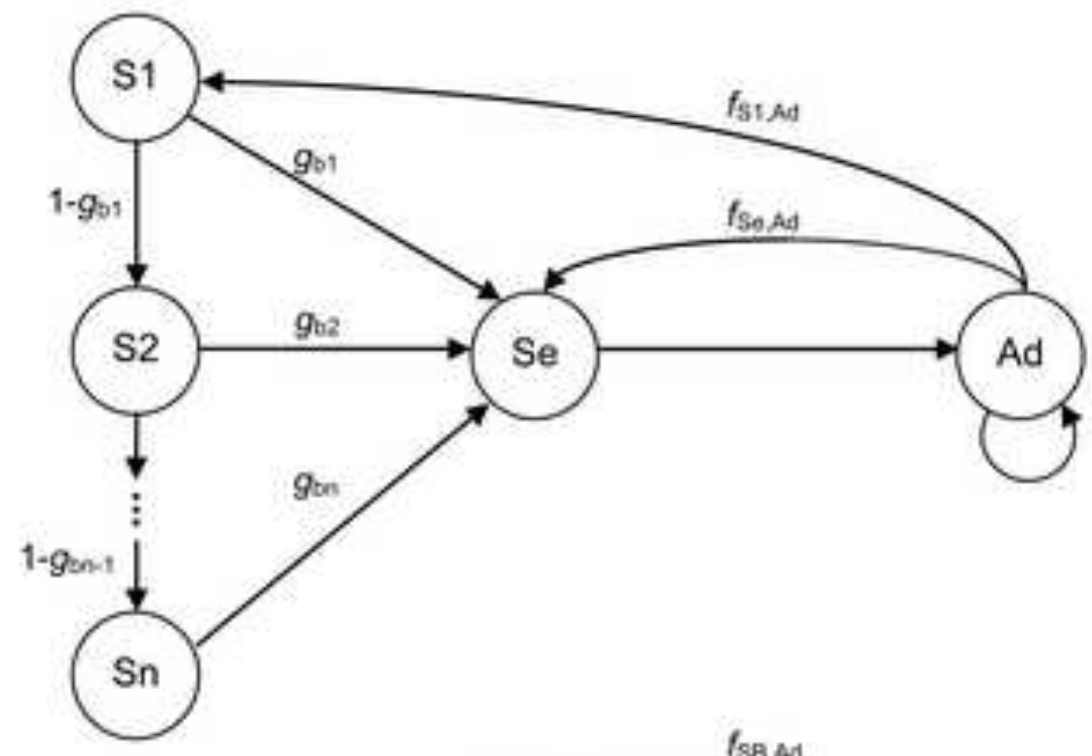

C

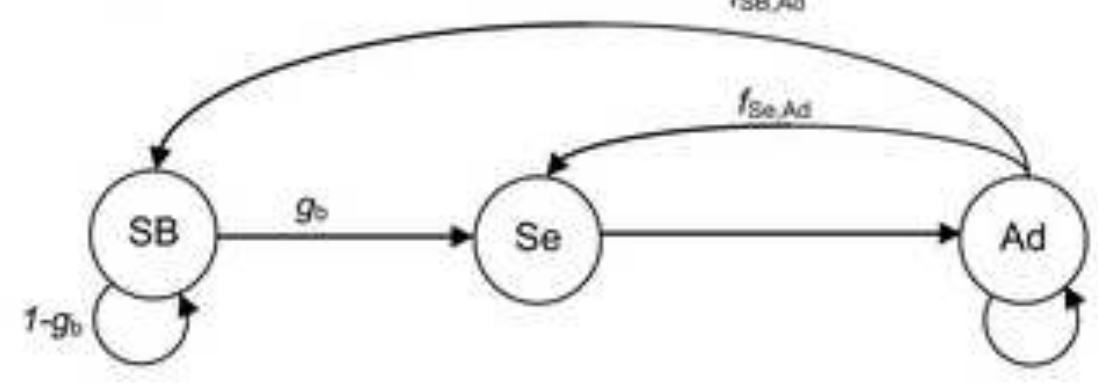

D

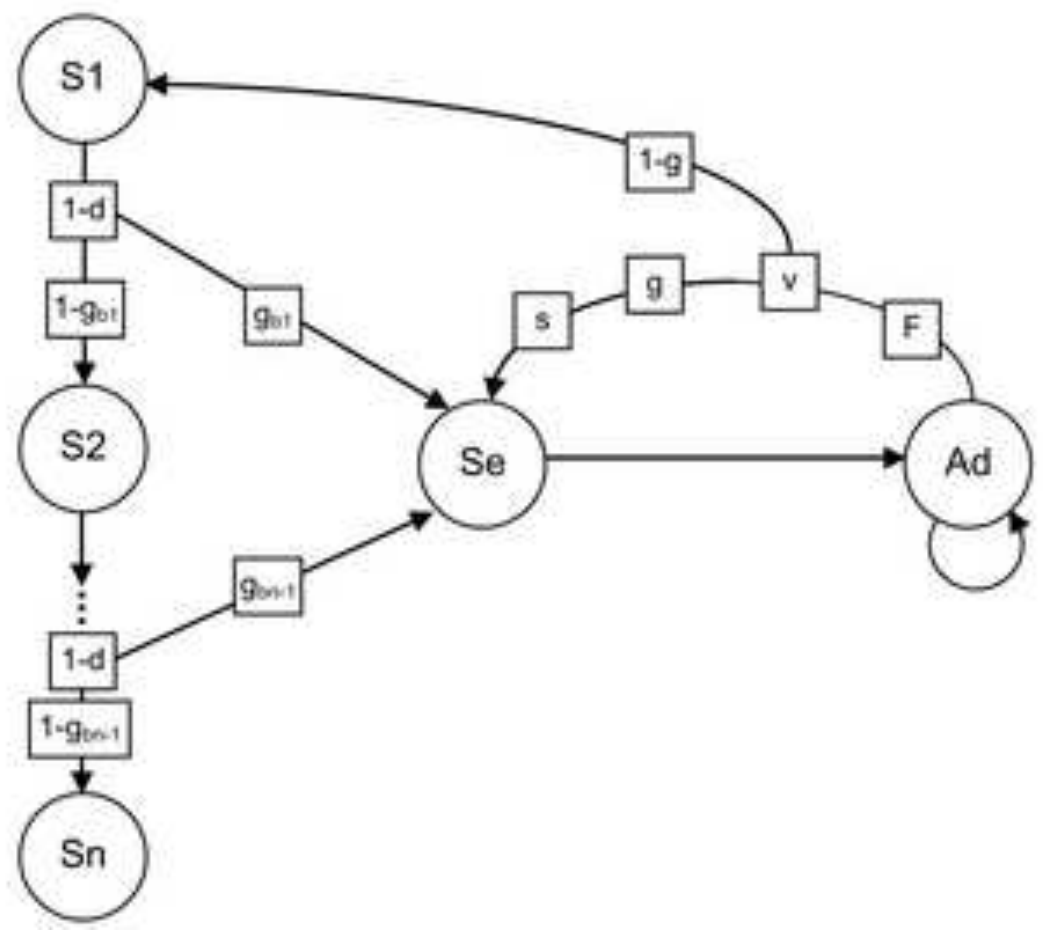


Figure

Click here to download high resolution image

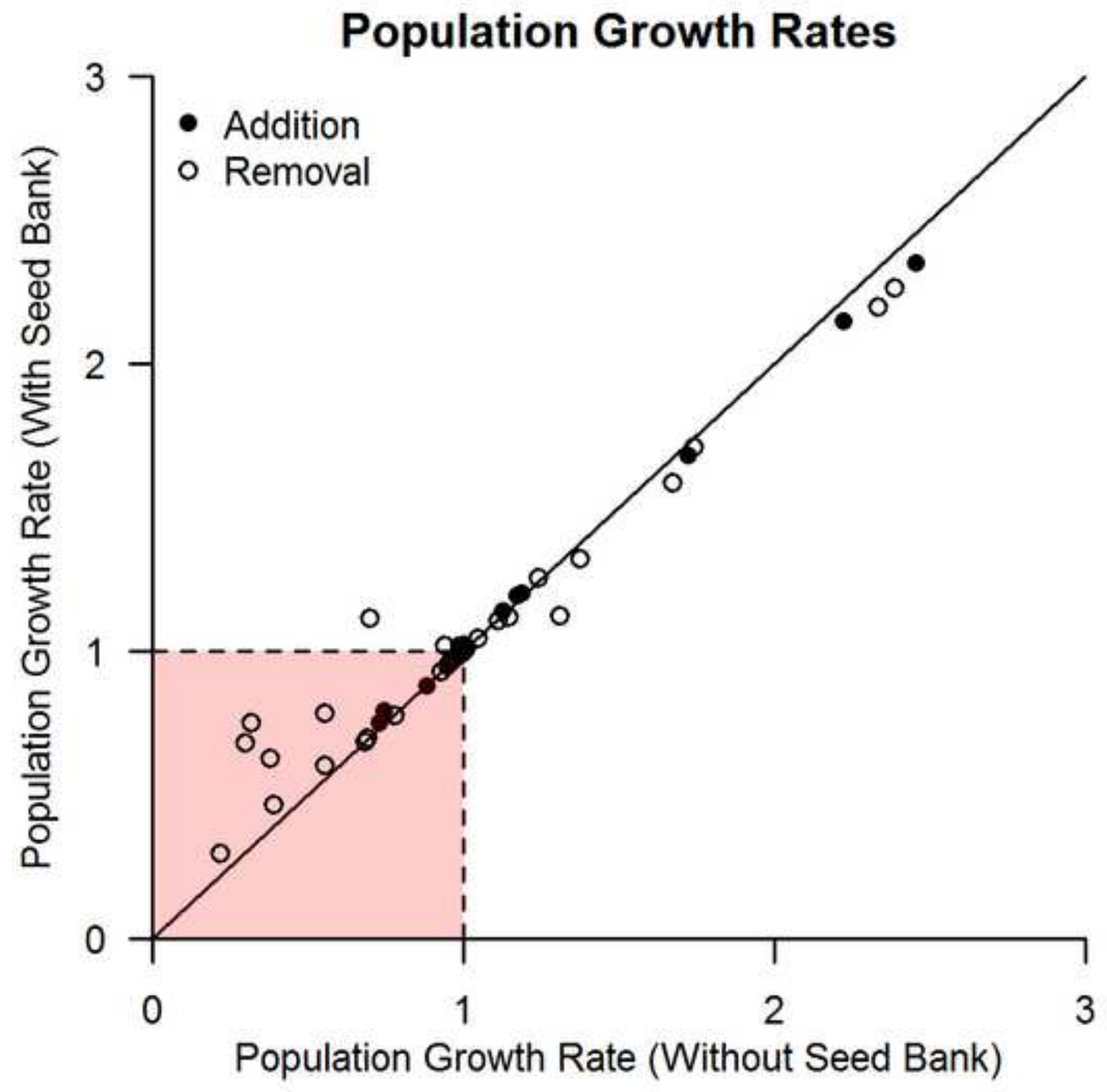


Uninformed priors
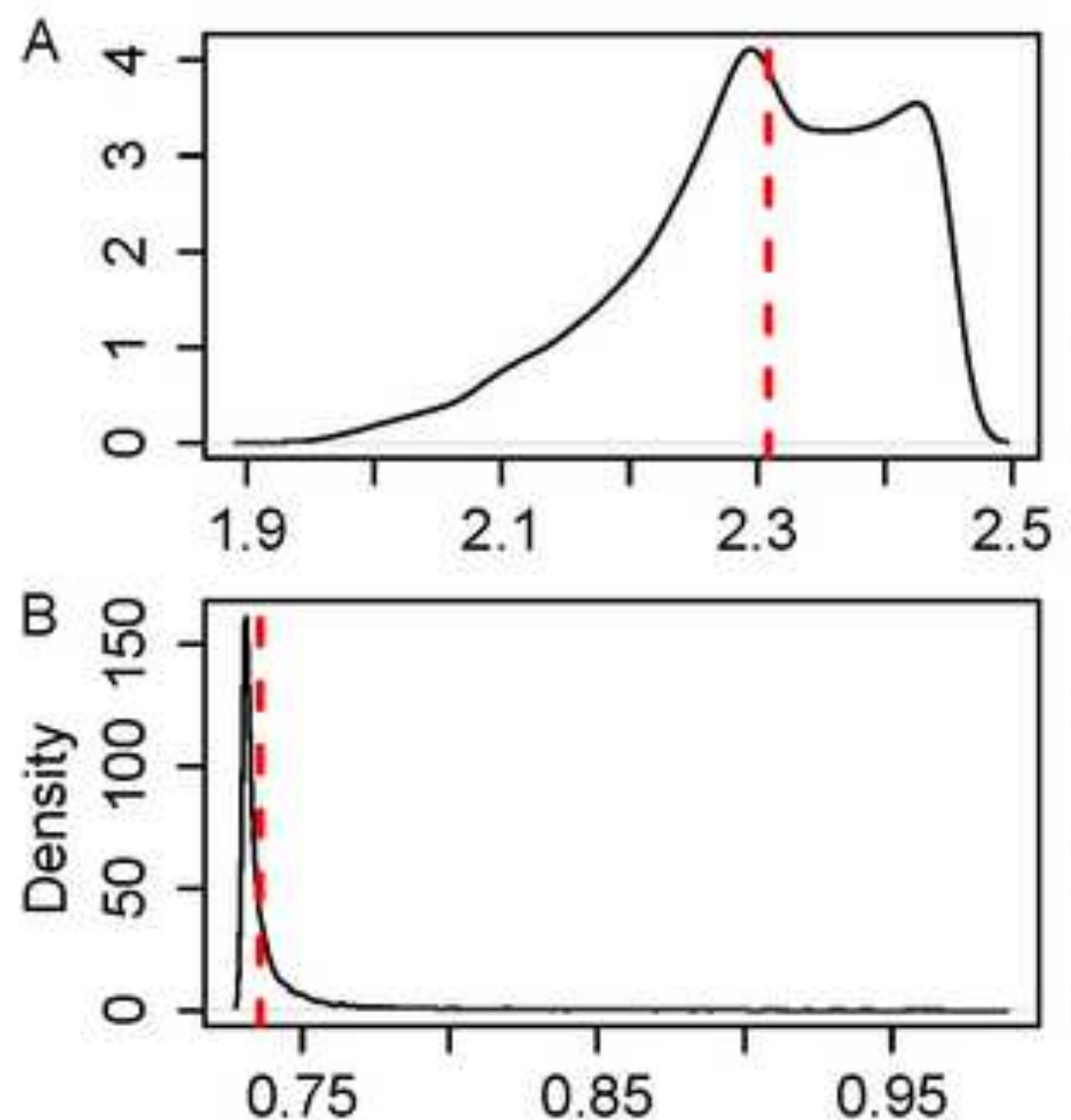

$\mathrm{C}$

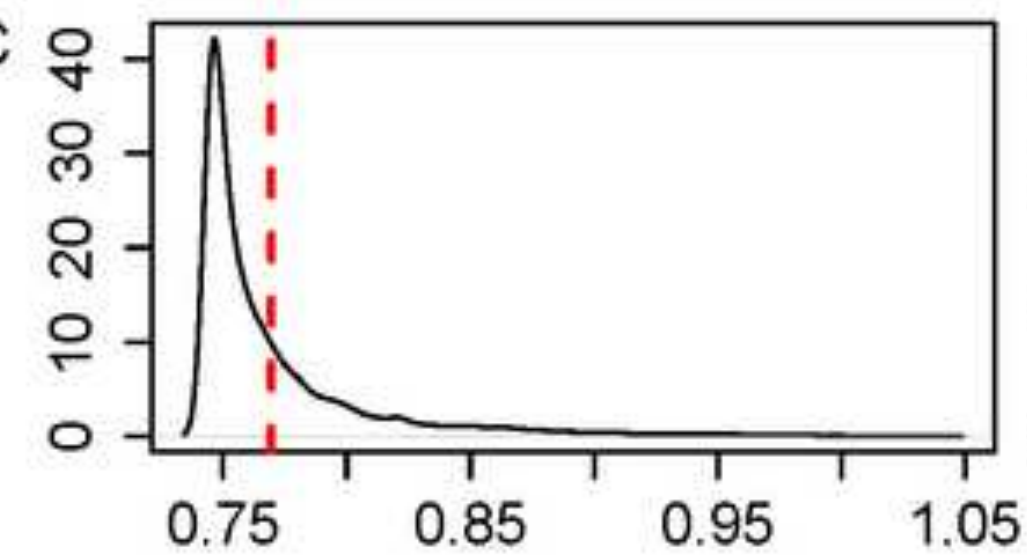

Informed priors
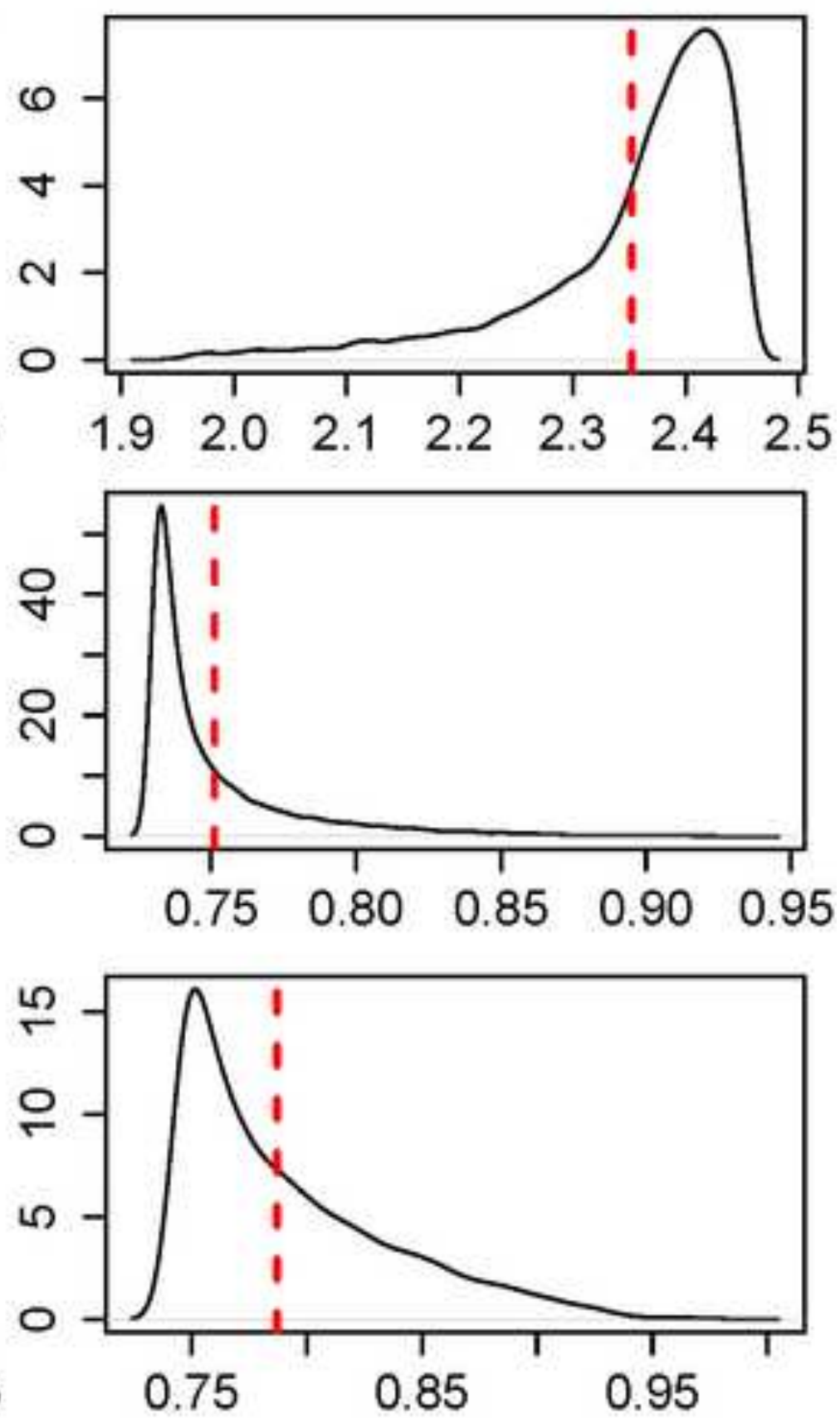

Population growth rate 

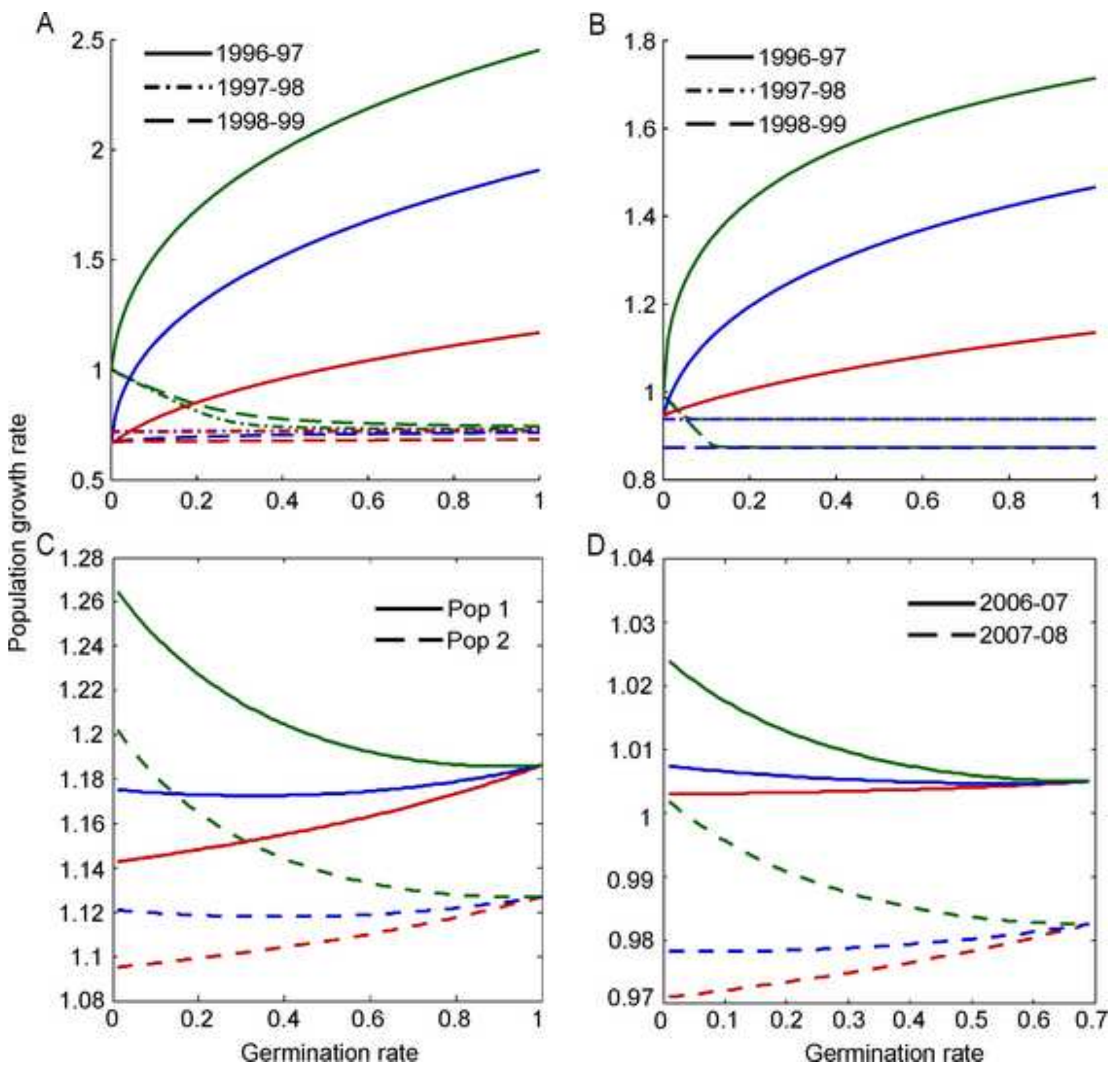


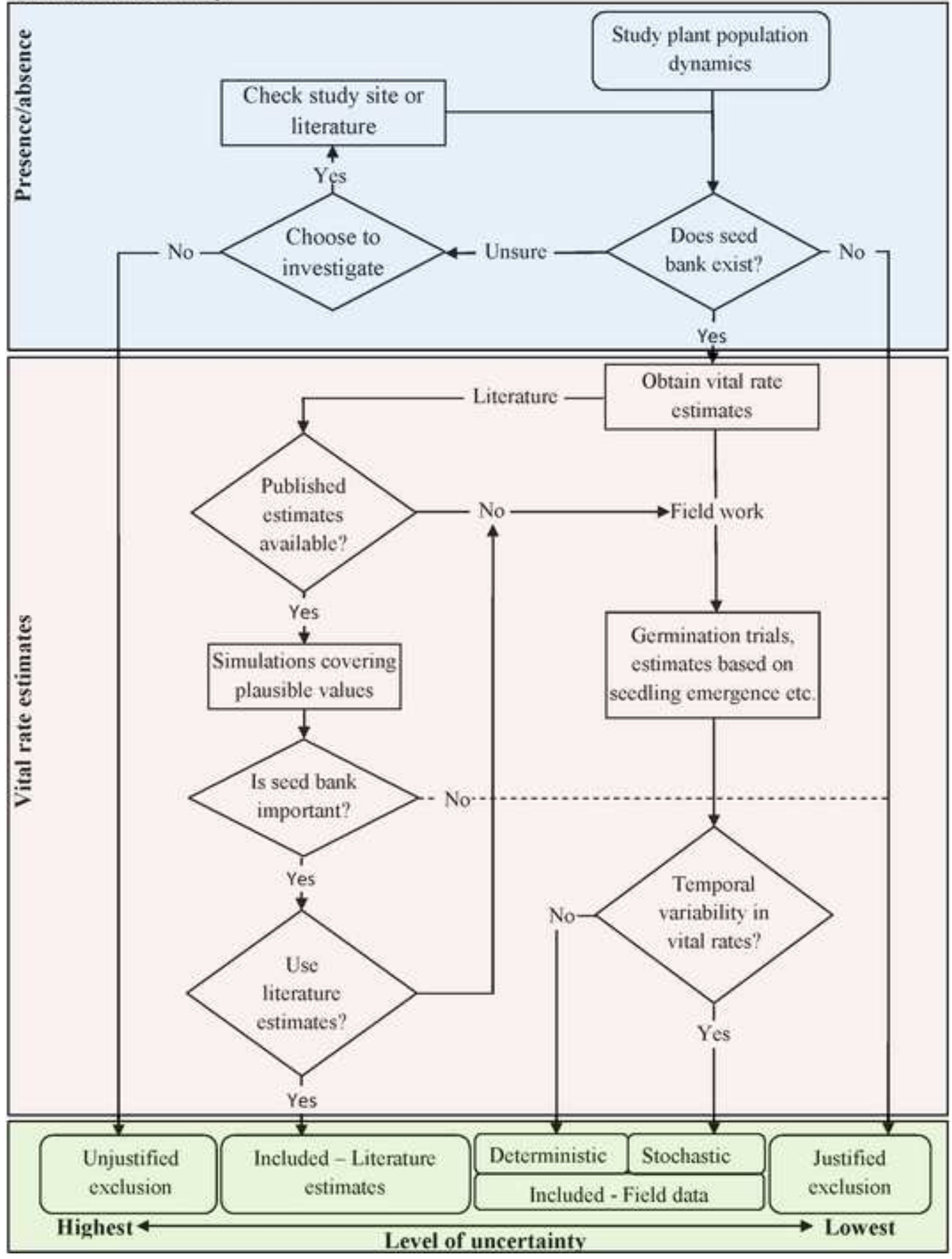



Supplementary material for online publication only
Click here to download Supplementary material for

Supplementary material for online publication only
Click here to download Supplementary material for online publication only: Sup Method S1.doc . to 

Supplementary material for online publication only
Click here to download Supplementary material for

Click here to download Supplementary material for online publication only: Sup Method S2.doc ention (1) 

Supplementary material for online publication only
Click here to download Supplementary material for

Click here to download Supplementary material for online publication only: Sup Table S1.doc . (1) (1) (a) thent

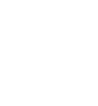

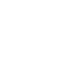
.

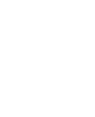
(1) (1) .

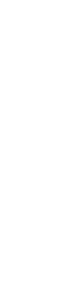

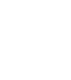

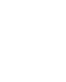

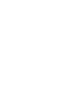

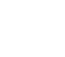

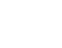

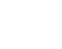

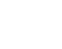

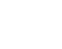

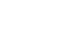

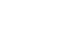

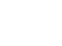

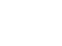

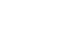

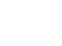



Supplementary material for online publication only
Click here to download Supplementary material for online publication only: Sup Table S2.doc

Supplementary material for online publication only
Click here to download Supplementary material for online publication only: Sup Table S2.docx Supplementary material for online publication only
Click here to download Supplementary material for online publication only: Sup Table S2.doc

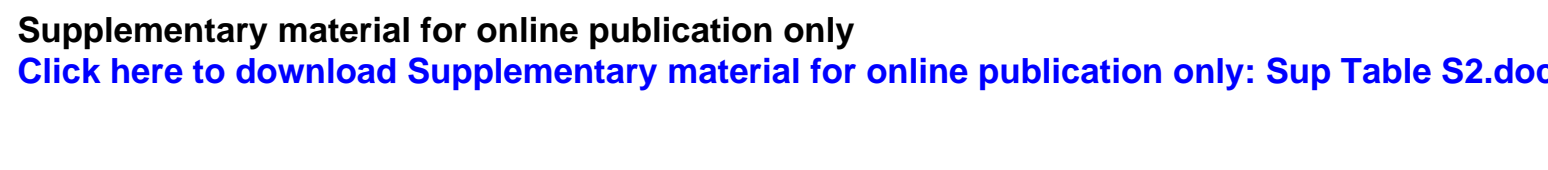

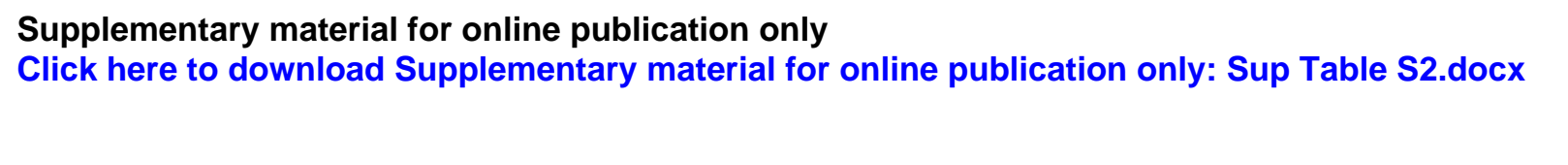

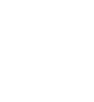

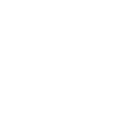

(1)

.

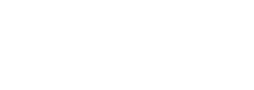

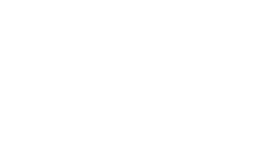
( . . . . . . . . . . . 列 

Supplementary material for online publication only
Click here to download Supplementary material for

Click here to download Supplementary material for online publication only: Sup Figure S1.docx

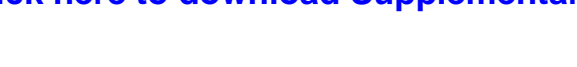
-

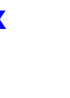

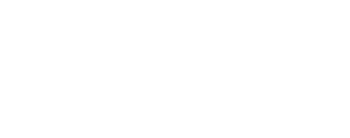
( (

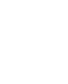

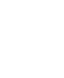

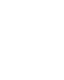

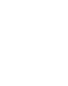
(1) (1) (1)

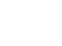

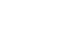

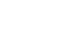

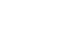

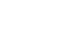

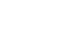

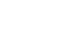

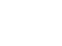

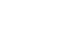

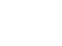



Supplementary material for online publication only
Click here to download Supplementary material for

Click here to download Supplementary material for online publication only: Sup Figure S2.docx
ck here to download Supplementary material for o

x

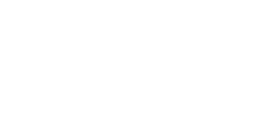


Credit Author Statement

VN contributed to the conceptualization, methodology, formal analysis, investigation and writing of the original draft, review and editing.

YB contributed to the conceptualisation, methodology and writing of the original draft, review and editing.

RSG contributed to the methodology and writing of the original draft, review and editing.

GW contributed to the conceptualisation, methodology, resources and writing of the original draft, review and editing. 